\title{
Comparing malaria surveillance with periodic spraying in the presence of insecticide-resistant mosquitoes: should we spray regularly or based on human infections?
}

\author{
Kevin E.M. Church* and Robert J. Smith?†
}

\section{Introduction}

It has been estimated that one in two humans who ever lived has been killed by malaria [7]. Three billion people — almost half the world's population - are at risk of malaria $[13,21,23]$. It is a leading cause of death and disease in many developing countries, where young children and pregnant women are the groups most affected. $40 \%$ of the world's population live in malaria-endemic areas [17]; $90 \%$ of deaths due to malaria occur in subSaharan Africa [18], 75\% of whom are African children [6]. In 2015, it caused more than 214 million acute illnesses and 438,000 deaths [27]. This represents a $37 \%$ reduction in cases over the previous 15 years [27].

This reduction has been largely driven by vector-control methods, promarily insecticide-treated bednets and indoor residual spraying (IRS) $[9,26]$; both are known to be highly effective [15]. The latter involves spraying houses and structures with insecticides, thereby killing mosquitoes after they have fed, in an effort to stop transmission of the disease. Recent data reconfirm the efficacy and effectiveness of IRS in malaria control in countries where it

\footnotetext{
*Department of Applied Mathematics, The University of Waterloo, Waterloo ON N2L 3G1 Canada

${ }^{\dagger}$ Department of Mathematics and Faculty of Medicine, The University of Ottawa, Ottawa ON K1N 6N5 Canada
} 
was implemented well [26]. Since many malaria vectors are endophilic, resting inside houses after taking a blood meal, they are particularly susceptible to be controlled through IRS. This method kills the mosquitoes after they have fed, thereby stopping transmission of the disease. The user is able to spray the whole house or dwelling on the inside, and under the eaves on the outside. The duration of effective action for timely, good-quality spraying is greater than six months [26].

Using these methods, malaria was eradicated or greatly reduced in many countries in the world between the 1940s and 1960s. Due to its success, DDT was rapidly introduced into public-health and malaria-control campaigns, and was the main insecticide used in the malaria-eradication campaign carried out between 1955 and 1969 [25]. There is evidence of resistance, but spraying with multiple insecticides has been successful in controlling Anopheles funestus, Anopheles gambiae and Anopheles melas in Equatorial Guinea, for example [20].

Surveillance is an important tool in disease management [10]; this is particularly true of malaria, which is spatially heterogeneous [2]. The World Health Organization has identified effective surveillance as a critical component in malaria elimination and has called for stronger surveillance systems to track and prevent outbreaks in endemic regions [27]. However, the majority of scientific and surveillance efforts are focused on countries that are unlikely to be the location of important emerging infectious diseases [10]. Furthermore, many countries with a high burden of malaria have weak surveillance systems and are not in a position to assess disease distribution and trends [27].

We assume that spraying occurs at times $t_{k}$. The effect of the insecticide is assumed to be instantaneous, resulting in a system of impulsive differential equations. Impulsive differential equations consist of a system of ordinary differential equations (ODEs), together with difference equations. Between impulses, the system is continuous, behaving as a system of ODEs. At the impulse points, there is an instantaneous change in state in some or all of the variables. This instantaneous change can occur when certain spatial, temporal or spatio-temporal conditions are met $[3,4,5,12]$. This is related to the use of pulse vaccinations [1], seasonal skipping in recurrent epidemics [24], antiretroviral drug treatment [16] and birth pulses in animals [19]. Impulse times may be fixed or non-fixed [22] and may be either timeor state-dependent.

This paper is organized as follows. In Section 2, we introduce the impul- 
sive model in its general form, detailing key assumptions. In Section 3, we develop preliminary results, such as existence and uniqueness properties. In Section 4 , we analyze a non-impulsive submodel consisting only of mosquito dynamics. In Section 5, we analyze the mosquito-only submodel with impulsive effects. In Section 6 we illustrate our theoretical results with numerical simulations. In Section 7, we determine global results for periodic orbits under a simplifying assumption. We conclude with a discussion and relegate all proofs to the appendix.

\section{The model}

All humans are either susceptible $(S)$, infected $(I)$ or partially immune $(R)$. Humans are born susceptible at a constant background birth rate $\pi$, independent of the population size. The background death rate is $\mu_{H}$. Susceptible humans can become infected after being bitten by infected mosquitoes at rate $\beta$. Infected humans die from the disease at rate $\gamma$, recover without immunity at rate $h$ or acquire immunity at rate $\alpha$. Humans with temporary immunity lose their immunity at rate $\delta$. All rates are per capita rates, unless otherwise mentioned.

In the absence of humans (and therefore new infections), mosquitoes undergo logistic growth with competition and a small probability of unidirectional mutation at birth. There is no competitive advantage or disadvantage to being infected with malaria. Malaria infection has a negligible effect on the lifespan of mosquitoes. Therefore, if $M_{w}$ and $M_{m}$ denote the population sizes of wild-type and mutant susceptible mosquitoes, $N_{w}$ and $N_{m}$ denote the corresponding infected mosquitoes, and $V_{w}=M_{w}+N_{w}$ and $V_{m}=M_{m}+N_{m}$ denote the total populations of wild-type and mutant mosquitoes (susceptible and infected), then we assume

$$
\begin{aligned}
\dot{M}_{w} & =\left((1-\epsilon) b_{w}-d_{w}\right) M_{w}+(1-\epsilon) b_{w} N_{w}, \\
\dot{M}_{m} & =\left(b_{m}-d_{m}\right) M_{m}+b_{m} N_{m}+\epsilon b_{w} M_{w}+\epsilon b_{w} N_{w}, \\
\dot{N}_{w} & =-d_{w} N_{w}, \\
\dot{N}_{m} & =-d_{m} N_{m},
\end{aligned}
$$


where $0<\epsilon \ll 1$ is the mutation rate. The birth and death rates are

$$
\begin{array}{ll}
b_{w}=b_{w}^{0}-K_{b w w} V_{w}-K_{b w m} V_{m}, & d_{w}=d_{w}^{0}+K_{d w w} V_{w}+K_{d w m} V_{m} \\
b_{m}=b_{m}^{0}-K_{b m m} V_{m}-K_{b m w} V_{w}, & d_{m}=d_{m}^{0}+K_{d m m} V_{m}+K_{d m w} V_{w}
\end{array}
$$

where all parameters are assumed to be positive. There is no vertical malaria transmission among mosquitoes. Notice that the above is not the usual, elegant definition of logistic growth. However, this parameter-heavy definition is necessary to take into account the correct mutation rate.

Wild-type mosquitoes are more evolutionarily fit in the absence of insecticide: Define the intrinsic growth rates

$$
r_{w}=b_{w}^{0}-d_{w}^{0}, \quad r_{m}=b_{m}^{0}-d_{m}^{0},
$$

carrying capacities

$$
K_{w}=\frac{r_{w}}{K_{b w w}+K_{d w w}}, \quad \quad K_{m}=\frac{r_{m}}{K_{b m m}+K_{d m m}},
$$

and competition coefficients

$$
\alpha_{w m}=\frac{K_{b w m}+K_{d w m}}{K_{b w w}+K_{d w w}}, \quad \quad \alpha_{m w}=\frac{K_{b m w}+K_{d m w}}{K_{b m m}+K_{d m m}} .
$$

It is assumed that these bulk parameters satisfy the inequalities

$$
r_{w} \geq r_{m}, \quad K_{w} \geq K_{m}, \quad \alpha_{m w} \geq \alpha_{w m},
$$

and at least one of the inequalities is strict.

Susceptible mosquitoes can become infected by biting an infectious human at rate $\beta_{M}$, which may depend on the sizes of human and mosquito subpopulations. The infection rate of humans by mosquitoes, $\beta$, is positively correlated to the population of infected mosquitoes. The infection rate of mosquitoes by humans, $\beta_{M}$, is positively correlated with the population of infected humans. Infection rates are assumed to be smooth functions of the population variables. All infection rates are nonnegative.

The probability of passive surveillance efforts detecting a given human malaria infection is given by $\eta$. Insecticide is sprayed if the number of reported malaria cases since the previous insecticide application reaches a critical level, $\bar{\Theta}$. Application of the insecticide instantaneously decreases the 
population of wild-type mosquitoes by a factor of $q \in(0,1)$. The insecticide has no effect on the mutant strain.

With these assumptions in place, we obtain the following system of impulsive differential equations:

$$
\begin{array}{rlrl}
\dot{S} & =\pi-\beta(P) S+h I+\delta R-\mu_{H} S, & & \Theta \neq \bar{\Theta} \\
\dot{I} & =\beta(P) S-h I-\alpha I-\left(\mu_{H}+\gamma\right) I, & & \Theta \neq \bar{\Theta} \\
\dot{R} & =\alpha I-\delta R-\mu_{H} R, & \Theta \neq \bar{\Theta} \\
\dot{M}_{w} & =\left((1-\epsilon) b_{w}-d_{w}\right) M_{w}+(1-\epsilon) b_{w} N_{w}-\epsilon b_{w} M_{w}-\beta_{M}(P) M_{w}, & \Theta \neq \bar{\Theta} \\
\dot{M}_{m} & =\left(b_{m}-d_{m}\right) M_{m}+\epsilon b_{w}\left(M_{w}+N_{w}\right)+b_{m} N_{m}-\beta_{M}(P) M_{m}, & & \Theta \neq \bar{\Theta} \\
\dot{N}_{w} & =\beta_{M}(P) M_{w}-d_{w} N_{w}, & & \Theta \neq \bar{\Theta} \\
\dot{N}_{m} & =\beta_{M}(P) M_{m}-d_{m} N_{m}, & & \Theta \neq \bar{\Theta} \\
\dot{\Theta} & =\eta \beta(P) S, & & \Theta \neq \bar{\Theta} \\
\Delta M_{w} & =-q M_{w}, & & \Theta=\bar{\Theta} \\
\Delta N_{w} & =-q N_{w}, & & \Theta=\bar{\Theta} \\
\Delta \Theta & =-\bar{\Theta}, & \Theta & =\bar{\Theta}
\end{array}
$$

Here $S, I$ and $R$ represent the number of susceptible, infected and temporarily immune humans, and $P=\left(S, I, R, M_{w}, N_{w}, M_{m}, N_{m}\right)$.

Due to the model assumptions, we may assume the infection rates satisfy

$$
\begin{array}{rlrl}
\frac{\partial \beta}{\partial N_{w}}>0, & \frac{\partial \beta}{\partial N_{m}} & >0, & \frac{\partial \beta_{M}}{\partial I}>0, \\
\beta\left(S, I, R, M_{w}, M_{m}, 0,0\right) & =\beta_{M}\left(S, 0, R, M_{w}, M_{m}, N_{w}, N_{m}\right)=0 .
\end{array}
$$

\section{Preliminary results}

\subsection{Existence and uniqueness of solutions and the bi- ological domain}

To properly discuss existence, uniqueness and boundedness of solutions of (5), it is necessary to adequately describe the "biological domain". Specifically, we must deal with the lack of positive invariance of the nonnegative cone. This lack of invariance is the result of incorporating mutation at birth 
with the logistic growth model. Indeed, it is possible for mosquito subpopulations to "become negative" if initial conditions are chosen improperly. A general invariance theorem is difficult to state; we provide a sufficient, implicit condition based on properties of a vector-only two-dimensional submodel. This submodel is considered in Sections 2-3, where a more explicit invariance theorem is given.

Theorem 1. Consider the two-dimensional system of ordinary differential equations,

$$
\begin{aligned}
& \dot{V}_{w}=r_{w} V_{w}\left(1-\frac{V_{w}+\alpha_{w m} V_{m}}{K_{w}}\right)-\epsilon V_{w}\left(b_{w}^{0}-K_{b w w} V_{w}-K_{b w m} V_{m}\right), \\
& \dot{V}_{m}=r_{m} V_{m}\left(1-\frac{V_{m}+\alpha_{m w} V_{w}}{K_{m}}\right)+\epsilon V_{w}\left(b_{w}^{0}-K_{b w w} V_{w}-K_{b w m} V_{m}\right) .
\end{aligned}
$$

Suppose the set

$$
\Omega_{b_{w}}=\left\{\left(V_{w}, V_{m}\right) \in \mathbb{R}_{+}^{2}: V_{w} \leq \frac{b_{w}^{0}-K_{b w m} V_{m}}{K_{b w w}}\right\}
$$

is positively invariant under the flow of (6). Then the set

$\Omega_{b_{w}}^{*}=\left\{(P, \Theta) \in \mathbb{R}_{+}^{8}: \Theta \leq \bar{\Theta}, S+I+R \leq \frac{\pi}{\mu_{H}},\left(M_{w}+N_{w}, M_{m}+N_{m}\right) \in \Omega_{b_{w}}\right\}$

is positively invariant under the flow of (5), and all solutions that begin in $\Omega_{b_{w}}^{*}$ exist for all positive time and are unique.

\subsection{Equivalence and stability of periodic solutions of fixed-time and autonomous models}

Let us denote $G=\left(S, I, R, M_{w}, M_{m}, N_{w}, N_{m}\right), \dot{G}=F(G)$ and $\Delta G=L G$, where the vector field $F$ and linear impulse $L$ are obtained from (5) by simply ignoring the $\Theta$ equations. Consider the following impulsive differential equation with impulses at fixed times $k T$, for integers $k$ and a fixed spraying period $T$ :

$$
\begin{array}{rlrl}
\dot{G} & =F(G), & t \neq k T, \\
\Delta G & =L G, & t & =k T .
\end{array}
$$


It is fairly obvious that periodic solutions of the autonomous model (5) give rise to periodic solutions of the model with fixed-time spraying events (7), provided the correct spraying period $T$ is chosen. It turns out that the converse of this statement holds as well. Additionally, certain uniqueness results are transferable. This equivalence of periodic orbits is applicable to many impulsive vector-control models, so we state and prove the following proposition in full generality. In the following, we assume that all differential equations in question admit unique, globally defined solutions.

Proposition 1. Consider the following impulsive differential equation undergoing impulse effects at fixed times $t_{k}=k T$ with $k \in \mathbb{Z}, T \in \mathbb{R}^{+}$and phase space $\Omega \times \mathbb{R}_{+}$, where $\Omega \subset \mathbb{R}_{+}^{n}$, the nonnegative orthant:

$$
\begin{aligned}
\frac{d x}{d t}=g(x), & t \neq k T \\
\Delta x & =a(x), \quad t=k T .
\end{aligned}
$$

Suppose (8) has a unique, non-trivial T-periodic solution with one impulse per cycle. Denote said periodic solution by $\varphi(t)$. Then, for any $u: \mathbb{R}^{n} \rightarrow \mathbb{R}$ satisfying $u(\varphi(t))>0$, the autonomous tracking system,

$$
\begin{array}{ll}
\frac{d x}{d t}=g(x), & \left.\Delta x\right|_{\theta=\bar{\theta}}=a\left(x^{-}\right), \\
\frac{d \theta}{d t}=u(x), & \left.\Delta \theta\right|_{\theta=\bar{\theta}}=-\bar{\theta},
\end{array}
$$

has a unique T-periodic solution up to phase shift, with one impulse per cycle, given by $(\varphi(t), \theta(t))$, whenever

$$
\bar{\theta}=\int_{0}^{T} u(\varphi(t)) d t
$$

Conversely, if (9) has a non-trivial $T_{0}$-periodic solution with one impulse per cycle for some $\bar{\theta} \in \mathbb{R}_{+}$, then (8) has a $T_{0}$-periodic solution when $T=T_{0}$. Moreover, $\bar{\theta}$ satisfies equation (10).

From now on, when referring to an autonomous model, uniqueness of periodic solutions will always be taken to mean uniqueness up to phase shift. The next lemma states that stability of periodic orbits in the system with impulses at fixed times is equivalent to the stability of the corresponding periodic orbit in the autonomous tracking system. 
Proposition 2. Let $\varphi(t)$ be a T-periodic solution of the system with fixed impulses (8) and let $\tilde{\varphi}(t)$ denote the corresponding periodic solution of the autonomous tracking system in (9). Suppose the solution operators of systems (8) and (9) are smooth with respect to initial conditions. Then $\varphi(t)$ is exponentially stable if and only if $\tilde{\varphi}(t)$ is orbitally asymptotically stable with asymptotic phase.

The previous two results clearly apply to our model (5) and the associated fixed-time model (7). As such, we have the following theorem.

Theorem 2. Suppose the model with spraying at fixed times, (7), has a Tperiodic solution $\varphi(t)$. If

$$
\bar{\Theta}=\int_{0}^{T} \eta \beta(P(\varphi)) S(\varphi) d t,
$$

then the function $\tilde{\varphi}(t)=(\varphi(t), \Theta(t))$ is a T-periodic solution of the autonomous model (5), where $\Theta(t)=\eta \beta(P(\varphi)) S(\varphi) . \varphi(t)$ is exponentially stable if and only if $\tilde{\varphi}(t)$ is orbitally asymptotically stable with asymptotic phase. If $\varphi(t)$ is the only T-periodic solution of $(7)$, then $\tilde{\varphi}(t)$ is the unique $T$-periodic solution of (5).

Corollary 2.1. Suppose the model with spraying at fixed times, (7), has a unique hyperbolic endemic periodic orbit, $\varphi(t ; T)$, for all $T \in\left(0, T^{+}\right)$for some $T^{+}>0$. Then, there exists $\bar{\Theta}^{+}>0$ such that, for all $\Theta \in\left(0, \bar{\Theta}^{+}\right)$, the autonomous model (5) has a periodic solution.

The above is largely a consequence of Theorem 2 and the Lebesgue dominated convergence theorem; it can be shown that the map

$$
T \mapsto \int_{0}^{T} \eta \beta(P(\varphi(t, T))) S(\varphi(t, T)) d t
$$

is continuous and vanishes at $T=0$ using the hyperbolicity assumption; the result follows immediately.

We can conclude that if the model with spraying at fixed times has a unique stable periodic solution of period $T$, then the autonomous tracking model does too, provided the spraying threshold $\bar{\Theta}$ is chosen appropriately. In a certain sense, the autonomous and fixed-time spraying strategies are "asymptotically equivalent" for initial conditions sufficiently close to the periodic orbit. However, we cannot rule out the existence of periodic solutions 
of the autonomous model (5) with a period that is different from $T$, and we know nothing of the global stability of the periodic solutions. Statements regarding these properties can be made for simplified versions of the aforementioned models, and this is considered in Section 4.

\subsection{Introducing the mosquito-only submodel}

To understand the dynamics of the full models (5) and (7), we first consider the two-dimensional system of impulsive differential equations with impulses at fixed times that describes the dynamics of the vector populations:

$$
\begin{array}{cc}
\dot{V}_{w}=r_{w} V_{w}\left(1-\frac{V_{w}+\alpha_{w m} V_{m}}{K_{w}}\right)-\epsilon V_{w}\left(b_{w}^{0}-K_{b w w} V_{w}-K_{b w m} V_{m}\right), & t \neq k T \\
\dot{V}_{m}=r_{m} V_{m}\left(1-\frac{V_{m}+\alpha_{m w} V_{w}}{K_{m}}\right)+\epsilon V_{w}\left(b_{w}^{0}-K_{b w w} V_{w}-K_{b w m} V_{m}\right), & t \neq k T \\
\Delta V_{w}=-q V_{w}, & t=k T .
\end{array}
$$

This system can be easily derived from the fixed-time model (7) by defining $V_{w}=M_{w}+N_{w}$ and $V_{m}=M_{m}+N_{m}$ and noticing that the derivatives and impulse conditions decouple from the human $(S, I, R)$ components. As previously illustrated in Theorem 2, not much information is lost by working with fixed-time spraying instead of autonomous spraying.

It turns out that a wealth of qualitatively different dynamics can be seen in this simplistic two-dimensional model (11). We will not attempt to classify all of them but will instead focus on the effect of adding the insecticide spraying in the case where the system without impulses exhibits one of two phase portraits: global stability of a coexistence equilibrium or bistability of a coexistence equilibrium with a mutant-only equilibrium. 


\section{Analysis of the mosquito-only submodel with- out impulses}

In this section, we consider the system (11) without impulses

$$
\begin{aligned}
& \dot{V}_{w}=r_{w} V_{w}\left(1-\frac{V_{w}+\alpha_{w m} V_{m}}{K_{w}}\right)-\epsilon V_{w}\left(b_{w}^{0}-K_{b w w} V_{w}-K_{b w m} V_{m}\right), \\
& \dot{V}_{m}=r_{m} V_{m}\left(1-\frac{V_{m}+\alpha_{m w} V_{w}}{K_{m}}\right)+\epsilon V_{w}\left(b_{w}^{0}-K_{b w w} V_{w}-K_{b w m} V_{m}\right) .
\end{aligned}
$$

\subsection{Nullcines}

Lemmas 1-2 characterize the $V_{m}$ nullcline. Lemma 3 describes the $V_{w}$ nullclines. Theorem 3 is a summary.

Lemma 1. Suppose at most one of the equalities

$$
\frac{b_{w}^{0} \alpha_{m w}}{K_{m}}=K_{b w w}, \quad \frac{b_{w}^{0}}{K_{m}}=K_{b w m}
$$

holds. There exists $\epsilon_{0}>0$ such that, for $0<\epsilon<\epsilon_{0}$, the $V_{m}$ nullcline is a non-degenerate hyperbola.

Lemma 2. Suppose the inequality

$$
\frac{K_{b w w}}{b_{w}^{0}} \leq \frac{\alpha_{m w}}{K_{m}}
$$

holds. There exists $\epsilon^{*}>0$ such that if $0<\epsilon<\epsilon^{*}$, then the conclusions of Lemma 1 hold, one branch of the hyperbola is nonpositive and intersects the origin, while the other branch intersects $\mathbb{R}_{+}^{2}$ in a curve and can be described by the graph of a strictly decreasing convex function $M: V_{m} \mapsto M\left(V_{m}\right)=V_{w}$. $M$ satisfies $M(0)=b_{w}^{0} / k_{b w w}$ and $M\left(K_{m}\right)=0$.

Lemma 3. The $V_{w}$ nullclines consist of the line $V_{w}=0$ and the parameterized line

$$
W\left(V_{m}, \epsilon\right)=-\left(\frac{\alpha_{w m} r_{w}-\epsilon K_{b w m} K_{w}}{r_{w}-\epsilon K_{b w w} K_{w}}\right) V_{m}+\frac{K_{w}\left(r_{w}-\epsilon b_{w}^{0}\right)}{r_{w}-\epsilon K_{b w w} K_{w}} .
$$

If $|\epsilon|$ is sufficiently small, $W(\cdot, \epsilon)$ is decreasing and $V_{w}(0, \epsilon)>0$. 
With the previous three Lemma in hand, we have the following qualitative description of the equilibrium points of the submodel (12).

Theorem 3. Suppose inequality (14) holds and at most one of the equalities of (13) is satisfied. There exists $\epsilon_{0}>0$ such that, for $0<\epsilon<\epsilon_{0}$, the mosquito-only submodel without impulses, (12), has the trivial extinction equilibrium $(0,0)$, in addition to exactly one of the following:

1. A mutant-only equilibrium, $M_{0}$.

2. A mutant-only equilibrium, $M_{0}$, and a single coexistence equilibrium, $E_{0}$.

3. A mutant-only equilibrium, $M_{0}$, and two coexistence equilibria, $C_{0}$ and $C_{1}$.

The mutant-only equilibrium has coordinates $\left(0, K_{m}\right)$, and the coexistence equilibria are formed by intersections of the nonnegative branch of the hyperbolic $V_{m}$ nullcline, $M$, with the parameterized $V_{w}$ nullcline, $W\left(V_{m}, \epsilon\right)$.

Though certainly possible, it will not be our goal to determine the stability of the equilibria in all of the above cases, nor to completely classify each case by constraints on the model parameters. One reason is that the resulting expressions are very complicated. Secondly, there are sufficient conditions that guarantee that, for example, Case 2 occurs, which will be discussed in Section 2.3. Finally, since $\epsilon$ will always be assumed to be small, it is much more beneficial to simply consider perturbations from $\epsilon=0$ by techniques of bifurcation theory, in the event we wish to study Case 3, which is the most difficult to classify. This will be the subject of Section 2.4. For the moment, we will briefly comment on Case 1.

In the subsequent sections, we will regularly make reference to the hyperbolic criteria; these will consist of the hypotheses of Theorem 3. Specifically, the hyperbolic criteria are satisfied if inequality (14) and at most one equality of (13) hold.

\subsection{The feasible and biologically relevant domains}

We first describe a nonnegative domain that is positively invariant, yet whose biological interpretation is inappropriate. 
Lemma 4. Suppose the hyperbolic criteria are satisfied and, additionally, that $b_{w}^{0}>K_{b w w} K_{w}$. Let $\gamma$ denote the backward orbit through the point $\left(b_{w}^{0} / K_{b w w}, 0\right)$. Let $\Omega$ be the domain in the nonnegative quadrant whose boundary consists of $\gamma$, the line segment connecting $\left(b_{w}^{0} / K_{b w w}, 0\right)$ to the origin and the positive $V_{m}$ axis. If $\epsilon>0$ is sufficiently small, $\Omega$ exists and is the largest positively invariant set contained in $\mathbb{R}_{+}^{2}$.

The domain defined in Lemma 4 is not biologically "correct", because the term $b_{w}=b_{w}^{0}-K_{b w w} V_{w}-K_{b w m} V_{m}$ appearing in the differential equation (12) represents the birth rate of wild-type mosquitoes, which should be nonnegative. Since the domain $\Omega$ described in Lemma 4 is unbounded, there are points in $\Omega$ where $b_{w}$ is negative. The most straightforward (though not necessarily optimal) way to fix this problem is as follows.

Theorem 4. Suppose the following inequalities hold, in addition to the hyperbolic criteria.

$$
b_{w}^{0}>K_{b w w} K_{w}, \quad \max \left\{\frac{K_{w}}{\alpha_{w m}}, K_{m}\right\}<\frac{b_{w}^{0}}{K_{b w m}} .
$$

Then, for $\epsilon>0$ sufficiently small, the set $\Omega_{b_{w}}$ of Theorem 1 is positively invariant under the flow of (12).

\subsection{The case of no coexistence equilibria}

In this section, we will justify our claim that the case of no coexistence equilibria is not of biological interest.

Proposition 3. Suppose the hypotheses of Lemma 4 are satisfied. If there are no coexistence equilibria, then the mutant-only equilibrium is globally attracting in $\Omega \backslash\{(0,0)\}$.

Therefore if there are no coexistence equilibria, the mutant-only equilibrium attracts all nonzero trajectories. Since it was assumed that the mutant strain of mosquito was evolutionarily weaker in the absense of insecticide than the wild-type, we can safely ignore this case.

\subsection{The case of a single coexistence equilibrium}

When $\epsilon$ is sufficiently small, so that Theorem 3 holds, there are two possible ways in which there can be only one coexistence equilibrium. The 
non-degenerate case is when the hyperbolic $V_{m}$ nullcline intersects the parameterized $V_{w}$ nullcline transversally; the degenerate case is where they intersect tangentially. Completely classifying the relationships between parameters in the degenerate case is tedious but certainly not impossible. For the non-degenerate case, we can provide the following classification when $\epsilon$ is small.

Theorem 5. Let the hyperbolic criteria be satisfied, and suppose $b_{w}^{0}>K_{b w w} K_{w}$. Then there exists $\epsilon_{0}>0$ such that, for $\epsilon<\epsilon_{0}$, there is a single coexistence equilibrium formed by the transversal intersections of two nullclines, provided one of the following holds:

A1.

$$
K_{m}<\frac{K_{w}}{\alpha_{w m}} \quad K_{m}<\alpha_{m w} K_{w}
$$

A2.

$$
K_{m}=\frac{K_{w}}{\alpha_{w m}}, \quad K_{m}<\alpha_{m w} K_{w}, \quad \alpha_{w m} b_{w}^{0}>K_{b w m} K_{w}, \quad \alpha_{m w}>1 .
$$

In this case, the coexistence equilibrium is globally asymptotically stable on $\mathbb{R}_{2}^{+} \backslash\left\{V_{w}=0\right\}$ if and only if the mutant-only equilibrium is unstable. This will be guaranteed if Condition A1 holds.

\subsection{The case of bistability and multiple coexistence equilibria}

In this section, we establish two conditions under which bistability can occur. In the first, bistability is already present when $\epsilon=0$, and it is preserved for $\epsilon>0$ small. In the second, the coexistence equilibrium is globally stable when $\epsilon=0$, and bistability occurs when $\epsilon>0$ is small, due to a bifurcation of the mutant-only equilibrium. In both cases, we demonstrate that a heteroclinic orbit exists, connecting the extinction equilibrium to one of the coexistence equilibria. This orbit is essentially the "bistability boundary".

Theorem 6 (Preservation of bistability). Suppose the inequalities

$$
K_{w}<\alpha_{w m} K_{m}, \quad K_{m}<\alpha_{m w} K_{w}, \quad 1<\alpha_{w m} \alpha_{m w}, \quad b_{w}^{0}>K_{b w w} K_{w}
$$


are satisfied, in addition to the hyperbolic criteria. Then there exists $\epsilon_{0}>$ 0 such that, for $0<\epsilon<\epsilon_{0}$, there is a pair of coexistence equilibria: the mutant-only equilibrium is a sink, and the extinction equilibrium is a saddle. There are no periodic orbits, and there is a heteroclinic orbit connecting the extinction equilibrium to the saddle coexistence equilibrium.

Theorem 7 (Bifurcation from wild-type-only global stability at $\epsilon=0$ ). Suppose the hyperbolic criteria are satisfied, in addition to the following.

B1. The inequalities

$$
\alpha_{m w} \alpha_{w m}>1, \quad b_{w}^{0}>K_{b w m} K_{m}, \quad K_{w} \neq r_{w}
$$

are satisfied.

B2. There exists a $C^{1}$ function $g: U \subset \mathbb{R} \rightarrow \mathbb{R}$, with $U$ an open set containing 0 , satisfying $g(0)=1$ and

$$
\frac{g^{\prime}(0)}{r_{w}-K_{w}}<\frac{\alpha_{m w} \alpha_{w m}}{K_{w} r_{w}\left(r_{w}-K_{w}\right)}\left(\frac{K_{w}\left(K_{b w m} r_{w}-K_{b w w} \alpha_{w m} r_{m}\right)}{r_{w}}+K_{b w w} K_{w}-b_{w}^{0}\right),
$$

where $K_{m}$ is a function of $\epsilon$ and can be written $K_{m}=\frac{K_{w}}{\alpha_{w m}} g(\epsilon)$.

Then there exists $\epsilon_{0}>0$ such that, for $0<\epsilon<\epsilon_{0}$, there is a pair of coexistence equilibria: the mutant-only equilibrium is a sink, and the extinction equilibrium is a saddle. There are no periodic orbits, and there is a heteroclinic orbit connecting the extinction equilibrium to the saddle coexistence equilibrium.

\section{Analysis of the mosquito-only submodel with impulse effects}

In this section, we investigate the effect of incorporating impulsive vector control on the mosquito-only submodel. We assume we are working in a feasible domain, such as the one described in Lemma 4.

To begin, we describe what happens to the extinction equilibrium at arbitrary (but small) mutation rates, as a function of spraying efficacy. 
Theorem 8 (Bifurcation at extinction). Define the quantity $q_{0}^{*}(\epsilon)$ as follows:

$$
q_{0}^{*}(\epsilon)=1-e^{-\left(r_{w}-\epsilon b_{w}^{0}\right) T} .
$$

If $\epsilon$ is sufficiently small, a biologically irrelevant (non-positive) periodic orbit collides with the extinction equilibrium, resulting in a transcritical bifurcation (in the one-dimensional centre dynamics) when $q=q_{0}^{*}(\epsilon)$. The extinction equilibrium transforms from a sink into a saddle, while the periodic orbit transforms from a saddle to a sink, as $q$ increases through $q_{0}^{*}(\epsilon)$. The periodic orbit remains biologically irrelevant for $q \approx q_{0}^{*}(\epsilon)$.

Next, we state the critical spraying threshold where the mutant-only equilibrium undergoes a saddle-node bifurcation.

Theorem 9 (Mutant-only saddle-node bifurcation). Define the quantity $q_{M}^{*}(\epsilon)$ as follows:

$$
\begin{aligned}
q_{M}^{*}(\epsilon) & =1-\exp \left\{T\left[\epsilon\left(b_{w}^{0}-K_{b w m} K_{m}\right)-c\right]\right\}, \\
c & =r_{w}\left(1-\frac{\alpha_{w m} K_{m}}{K_{w}}\right) .
\end{aligned}
$$

Suppose the inequality

$$
\frac{r_{m}\left(e^{c T}-e^{-r_{m} T}\right)}{\alpha_{w m}\left(r_{m}+c\right) T}<e^{c T} \int_{0}^{T} e^{c s}\left[1+\frac{\alpha_{w m} \alpha_{m w} r_{m}}{r_{m}+c}\left(e^{c s}-e^{-r_{m} s}\right)\right] d s
$$

holds. Then, for $\epsilon$ sufficiently small, we have $0<q_{M}^{*}(\epsilon)<1$, and the mutantonly equilibrium for system (11) undergoes a saddle-node bifurcation at parameter $q=q_{M}^{*}(\epsilon)$. Specifically, a locally stable, nonnegative periodic orbit collides with the mutant-only equilibrium, losing stability and becoming nonpositive, while the mutant-only equilibrium becomes locally stable, as $q$ increases through $q_{M}^{*}(\epsilon)$.

When $\epsilon=0$, the wild-type equilibrium is replaced with a wild-type periodic orbit, when $q>0$ (that is, when the impulse effect is included). An explicit formula for this periodic orbit for $t \in(0, T]$ is as follows:

$$
\begin{aligned}
\tilde{w}(t ; q) & =\frac{K_{w} e^{r_{w} t} w_{0}(q)}{K_{w}+\left(e^{r_{w} t}-1\right) w_{0}(q)}, \\
w_{0}(q) & =\frac{K_{w}\left(e^{r_{w} T}(1-q)-1\right)}{e^{r_{w} T}-1} .
\end{aligned}
$$


It is worthwhile determining the critical value of $q>0$ at which the wildtype periodic orbit loses its stability when there is no mutation (that is, when $\epsilon=0)$. There are two critical control efficacies.

Proposition 4 (Critical control efficacies pertinent to stability of the wildtype-only periodic orbit with no mutation). Define the quantities $q_{1}^{*}$ and $q_{2}^{*}$ as follows:

$$
\begin{aligned}
& q_{1}^{*}=1-\exp \left(-r_{w} T\right) \\
& q_{2}^{*}=1-\exp \left(-r_{w} T\left(1-\frac{K_{m}}{\alpha_{m w} K_{w}}\right)\right) .
\end{aligned}
$$

The wild-type-only periodic orbit for system (11) with no mutation $(\epsilon=0)$ undergoes a transcritical bifurcation, colliding with the the extinction equilibrium, losing stability and becoming nonpositive, as the parameter $q$ increases through $q_{1}^{*}$, while the extinction equilibrium becomes a saddle point. The linearization of the wild-type-only periodic orbit has a simple unit eigenvalue when $q=q_{2}^{*}$, and this periodic orbit loses stability as $q$ increases through $q_{2}^{*}$. The inequality $0<q_{2}^{*}<q_{1}^{*}<1$ holds, provided $K_{m}<\alpha_{m w} K_{w}$.

We do not prove the above proposition, since its correctness can be inferred from the proof of the following theorem, which states that, under certain genericity assumptions, there is a bifurcation curve $c(q)=(V(q), \epsilon(q))$, defined in a neighbourhood of $q_{2}^{*}$, satisfying $c\left(q_{2}^{*}\right)=\left(w_{0}\left(q_{2}^{*}\right), 0\right)$ and for which $V(q)$ is corresponds to a periodic orbit of system (11) with spraying efficacy $q$ and mutation rate $\epsilon(q)$.

Theorem 10 (Existence of a bifurcation curve near the wild-type-only periodic orbit with no mutation). Suppose the following inequality is satisfied:

$$
\begin{aligned}
& \frac{e^{r_{m} T} r_{w} \alpha_{w m}}{e^{r_{w} T}\left(e^{r_{w} T}-1\right)\left(1-q_{2}^{*}\right)^{2}}\left(\frac{e^{r_{w} T}\left(1-q_{2}^{*}\right) \cdot\left(e^{r_{w} T}\left(1-q_{2}^{*}\right)-1\right)}{q}\right)^{\frac{r_{m} \alpha_{m} w}{r_{w} K_{m}}} \times \\
& F \times\left(\left(1-q_{2}^{*}\right) \frac{r_{w} \alpha_{w m}}{r_{m} K_{w}}\left(e^{r_{m} T}-1\right)-\frac{q_{1}^{*}}{K_{w}}\right)-\frac{2}{K_{w}}\left(e^{r_{m} T}-1\right) \neq 0,
\end{aligned}
$$

where

$$
F={ }_{2} F_{1}\left(\frac{r_{m} \alpha_{m w}}{r_{w} K_{m}}+3, \frac{r_{m}}{r_{w}} ; \frac{r_{m}+r_{w}}{r_{w}} ; \frac{e^{r_{w} T}\left(1-q_{2}^{*}\right)-1}{q_{2}^{*}}\right),
$$

and ${ }_{2} F_{1}$ is the Gauss hpergeometric function. Define $N(V, q, \epsilon)=\phi(T ; V, q, \epsilon)-$ $V$, where $t \mapsto \phi(t ; V, q, \epsilon)$ is the solution map of (11) with initial condition 
$\phi(0 ; V, q, \epsilon)=V$, initialized from time $t=0$ in the model time coordinates. There exists a unique $C^{1}$ curve $c: q \mapsto(V(q), \epsilon(q))$, defined in a neighbourhood $N$ of $q_{2}^{*}$, with the following properties.

1. The function $c=(V, \epsilon)$ satisfies the equalities $V\left(q_{2}^{*}\right)=w_{0}\left(q_{2}^{*}\right), \epsilon\left(q_{2}^{*}\right)=$ $0, \frac{\partial \epsilon}{\partial q}\left(q_{2}^{*}\right)=0$.

2. $N(V(q), \epsilon(q), q)=0$ for $q \in N$. That is, $V(q)$ corresponds to the initial condition at time $t=0$ of a periodic solution of system (11) with mutation rate $\epsilon(q)$ and spraying efficacy $q$.

3. $D_{V} N(V(q), \epsilon(q), q)$ is non-invertible for $q \in N$.

\section{Numerical Simulations}

In this section, we provide graphical representations of the bifurcations that can occur in the mosquito-only submodel by simulating the model numerically. We also approximate the relative sizes of the basins of attraction for the various equilibria and periodic orbits of the model, under the assumption that, in the absence of insecticide, the model exhibits bistability. The results suggest that the basin of attraction of the mutant-only state increases as the efficacy of spraying increases, and that another bifurcation may occur involving two coexistence periodic orbits. This is further supported by a readily obtained analytical lower bound on the size of the basin of attraction.

In Figure 1, plots are displayed of solutions curves with two different intial conditions: $(10,10)$ and $(10,90)$, for initial populations of wild-type and mutant mosquitoes, respectively. The curve from the smaller initial condition approaches a coexistence state, while the curve from the initial condition with a large initial mutant population approaches the mutant-only equilibrium. This is as predicted by Theorem 6; one can verify that the illustrative parameters satisfy the bistability conditions.

When the spraying is included on a quarterly basis $(T=365 / 4)$ with a mild efficacy $(q=0.2)$, the bistability is preserved. This is shown in Figure 2, in which different initial conditions clearly yield qualitively different results when the differential equations are simulated. These trajectories persist with perturbed initial conditions.

The size of basin of attraction of the mutant-only equilibrium is essentially constant, up until $q \approx 0.7495$. At this point, another bifurcation occurs; the 

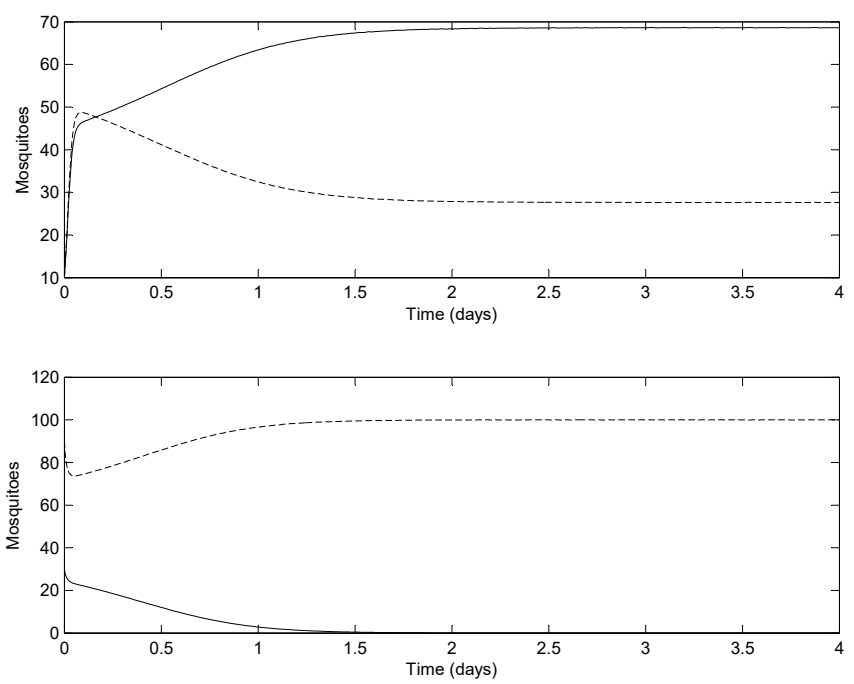

Figure 1: Plots of wild-type (solid line) and mutant (dashed line) mosquito populations for four days, with no spraying $(q=0)$. Illustrative parameters are chosen as $b_{w}^{0}=100, r_{w}=80, r_{m}=75, K_{b w w}=0.5, K_{d w w}=0.5$, $K_{w}=100, K_{m}=100, \alpha_{w m}=1, \alpha_{m w}=1.2$ and $\epsilon=0.05$. Top: initial condition $(10,10)$. Bottom: initial condition $(10,90)$. Initial conditions are ordered pairs of initial susceptible and infected populations.

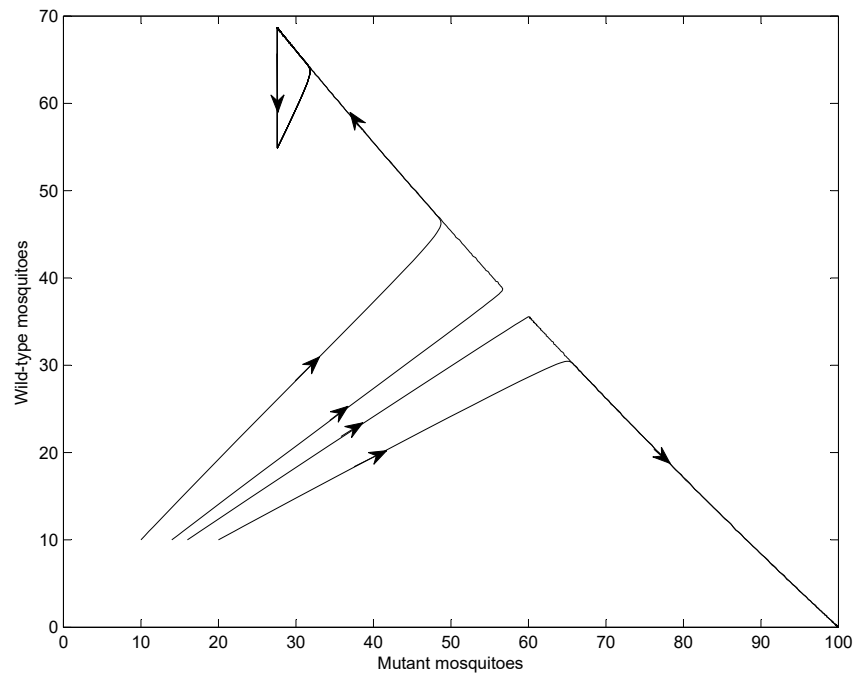

Figure 2: Plots of mosquito populations in the phase plane with spraying efficacy $q=0.2$ and spraying period $T=0.25 \times 365$ days; arrows indicate direction of forward time. Four initial conditions are chosen on the line $V_{w}=10$; notice that half of the chosen solutions converge to a coexistence periodic orbit, while the others converge to the mutant-only equilibrium. 


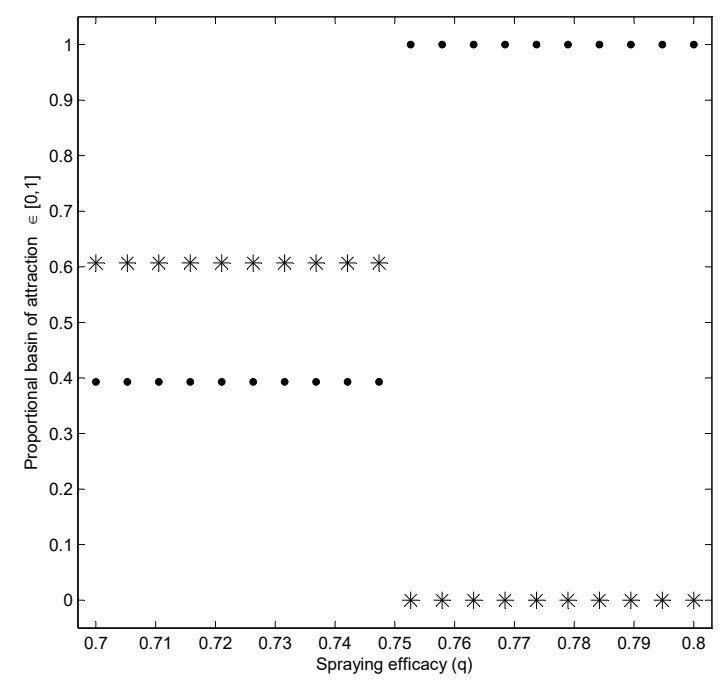

Figure 3: Proportion of trajectories in the set $U=\left\{0 \leq V_{w}+V_{m}<110\right\}$ that converge to the mutant-only equilibrium (dots) and the stable coexistence equilibrium (stars), as a function of spraying efficacy, $q$, ranging from 0.7 to 0.8. Initial conditions of the form $X(0)=X\left(0^{+}\right)$were used for this figure. Notice that the proportions, and hence basins of attraction, appear numerically constant, until the mutant-only equilibrium begins attracting almost all points in $U$ at approximately $q=\tilde{q} \approx 0.7406$. The computations required to produce this figure are numerically expensive; the (somewhat stiff) vector field is integrated more than 4000 times to produce only twenty samples for $q$ in the interval $[0.7,0,8]$. 
mutant-only equilibrium abruptly becomes globally stable. See Figure 3. This change in stability has nothing to do with a bifurcation of the mutantonly equilibrium, since that equilibrium is locally stable and hyperbolic for all $q \in[0,1]$. We conjecture that, at some critical spraying efficacy, $\tilde{q}$, the stable coexistence periodic orbit collides with an unstable coexistence periodic orbit, with the result being that they annihilate; that is, neither orbit persists for $q>\tilde{q}$. This is certainly possible, for there is indeed an unstable coexistence equilibrium present when $q=0$, which lies on the bistability boundary. This equilibrium generically persists for $q$ sufficiently close to $q=0$, as a periodic orbit. It should be mentioned that the size of the basin of attraction depends on the initial time coordinate, and in this case, initial conditions of the form $X(0)=X\left(0^{+}\right)$were used. This means that spraying does not occur at time $t=0$, but only begins at time $T$

The presence of such a bifurcation is more difficult to detect analytically, however. If the unstable coexistence equilibrium is hyperbolic when $q=$ 0 , then the bifurcation cannot be analytically detected using only a local analysis. If the associated equilibrium is non-hyperbolic when $q=0$, then it likely coincides with the other coexistence equilibrium. When $\epsilon=0$, a codimension-two bifurcation can occur at this equilibrium for a specific value of $q$, as illustrated by Theorem 10, which would complicate the analysis. Conversely, when $q=0$ and $\epsilon \neq 0$, it is difficult to analytically express the relevant equilibrium point.

It should be mentioned that the conclusions on the size of the basin of attraction of the above numerical simulations will differ if initial conditions of the form $X(0)=X\left(0^{-}\right)$are used instead. However, with the initial conditions as chosen, beginning spraying at time $t=T$ may be a more biologically appropriate interpretation, since in reality, one would expect the mosquito population to be closer to its natural coexistence equilibrium than to, say, the extinction equilibrium. Allowing $T$ units of time to pass before beginning to spray allows for the "pre-calibration" of the system.

For the sake of mathematical precision, we will discuss what may be observed if spraying begins at time $t=0$. From the phase portrait implied by Theorem 6, the stable coexistence equilibrium is separated from the mutant-only equilibrium by a heteroclinic orbit through the extinction state and another, unstable coexistence equilibrium. The heteroclinic orbit can be interpreted as the stable manifold of the "middle" unstable coexistence equilibrium. Due to the orientation of the nullclines, the global stable manifold, restricted to the positive quadrant, can be identified with a single 
smooth function, $b\left(V_{m}\right)$. It follows that when $q=0$, the basin of attraction of the mutant-only equilibrium, denoted $M_{0}$, contains the interior of the region bounded by the curves $V_{w}=0$ and $V_{w}=b\left(V_{m}\right)$.

Now, if $q>0$, any initial condition $X=\left(V_{m}, V_{w}\right)$ satisfying the inequality $V_{w}(1-q)<b\left(V_{m}\right)$ will be mapped immediately mapped into the (continuous) basin of attraction of $M_{0}$ at time $t=0^{+}$. As such, all initial conditions lying below the curve $V_{w}=\frac{b\left(V_{m}\right)}{1-q}$ lie in the (impulsive) basin of attraction. If we denote $B_{q}$ to be (impulsive) basin of attraction of $M_{0}$ at spraying efficacy $q$, the previous discussion implies that $B_{q}$ is at least $\frac{q}{1-q} \%$ larger than $B_{0}$, in the sense that

$$
\frac{\mu\left(B_{q}\right)}{\mu\left(B_{0}\right)} \geq \frac{\int_{0}^{V_{m}^{+}} \frac{b(m)}{1-q} d m}{\int_{0}^{V_{m}^{+}} b(m) d m}=1+\frac{q}{1-q}
$$

and $V_{m}^{+}$is any given prescribed upper bound on the number of mutant mosquitoes.

The above analysis provides another possible explanation of the theorized, numerically-motivated bifurcation discused earlier. The boundary of the impulsive basin of attraction of $M_{0}$ dominates a function that is everywhere monotone increasing in $q$, so it seems reasonable that the true boundary may interact with the stable coexistence periodic orbit at some critical value of $q$, resulting in a bifurcation. This also serves to explain the appeared constancy of the basin of attraction exhibited in the numerical simulations, prior to the bifurcation point. The growth of the impulsive basin of attraction of $M_{0}$ might not have been observed because the stable coexistence periodic orbit attracted points outside of the continuous basin of attraction of $M_{0}$ very quickly. Solutions numerically converged to the periodic orbit within the time interval $(0, T)$ and then, until $q \approx 0.7406$, the action of the impulse failed to bring solutions across the basin boundary, let alone the lower boundary, $V_{w}=\frac{b\left(V_{m}\right)}{1-q}$.

\section{Global existence and uniqueness results for periodic orbits in a simplified model}

The model from Section 1 can be simplified - and much more information obtained about the nature of its solutions — if we make the simplifying 
assumption that there are no mutant mosquitoes and that all mosquitoes are infectious. That is, we set $M_{m}=N_{m}=\epsilon=0$ and define $\Psi=M_{w}+N_{w}$, so that $\Psi^{\prime}=r_{w}\left(1-\frac{\Psi}{K_{w}}\right)$. Under this assumption, we can say much more about the endemic periodic orbits. The impulsive differential equations are

$$
\begin{aligned}
\dot{S} & =\pi-\beta(P) S+h I+\delta R-\mu_{H} S, & & t \neq k T, \\
\dot{I} & =\beta(P) S-h I-\alpha I-\left(\mu_{H}+\gamma\right) I, & & t \neq k T, \\
\dot{R} & =\alpha I-\delta R-\mu_{H} R, & & t \neq k T, \\
\dot{\Psi} & =r_{w} \Psi\left(1-\frac{\Psi}{K_{w}}\right), & & t \neq k T, \\
\Delta \Psi & =-q \Psi, & & t=k T,
\end{aligned}
$$

\subsection{Existence and stability of periodic orbits for the system with impulses at fixed times}

In contrast to the full model, the reduced model with spraying at fixed times is much more amenable to analytical techniques. Our first result pertains to the existence of periodic orbits under the assumption that the transmission rate is a linear function of the mosquito population (i.e., mass-action transmission).

Lemma 5. Suppose $\beta(P)=\beta_{H} \Psi$, for some $\beta_{H} \in \mathbb{R}_{+}$. Then the system with impulses at fixed times (21) has a nontrivial, nonnegative periodic solution, provided $T>T_{*} \equiv-\frac{\log (1-q)}{r_{w}}$. If $T \leq T^{*}$, the disease-free equilibrium is a global attractor.

A similar result can be obtained for standard incidence, with the caveat that we must assume that the death rate due to malaria is absent.

Lemma 6. Suppose $\beta(P)=\beta_{H} \frac{\Psi}{S+I+R}$, and $\gamma=0$. Then the system with impulses at fixed times (21) has a nontrivial, nonnegative periodic solution provided $T>T_{*} \equiv-\frac{\log (1-q)}{r_{w}}$. If $T \leq T_{*}$, the disease-free equilibrium is a global attractor.

The final result of this section is a perturbation result, applicable to either mass-action or standard-incidence transmission. It states that if certain parameters are sufficiently small, a unique, positive, exponentially stable periodic orbit exists. 
Theorem 11. There exist $\epsilon_{1}, \ldots, \epsilon_{4}>0$ such that, for

$$
\gamma<\epsilon_{1}, \quad \delta<\epsilon_{2}, \quad h<\epsilon_{3}, \quad \alpha<\epsilon_{4}
$$

the system with impulses at fixed times (21) has a unique, nonnegative, hyperbolic periodic solution, provided either $\beta(P)=\beta_{H} \Psi$ or $\beta(P)=\beta_{H} \frac{\Psi}{S+I+R}$ and $T>T_{*}$, where $T_{*}$ is defined as in Lemma 6 . If $T>T_{*}$, the periodic solution is exponentially stable.

\subsection{Properties of periodic orbits in the autonomous tracking model}

We will now characterize the periodic orbits of the simplified autonomous tracking model,

$$
\begin{aligned}
\dot{S} & =\pi-\beta(P) S+h I+\delta R-\mu_{H} S, & & \Theta \neq \bar{\Theta}, \\
\dot{I} & =\beta(P) S-h I-\alpha I-\left(\mu_{H}+\gamma\right) I, & & \Theta \neq \bar{\Theta}, \\
\dot{R} & =\alpha I-\delta R-\mu_{H} R, & & \Theta \neq \bar{\Theta}, \\
\dot{\Psi} & =r_{w} \Psi\left(1-\frac{\Psi}{K_{w}}\right), & & \Theta \neq \bar{\Theta}, \\
\dot{\Theta} & =\eta \beta(P) S, & & \Theta \neq \bar{\Theta}, \\
\Delta \Psi & =-q \Psi, & & \Theta=\bar{\Theta}, \\
\Delta \Theta & =-\bar{\Theta}, & & \Theta=\bar{\Theta} .
\end{aligned}
$$

Theorem 12. Suppose one of the following conditions is satisfied.

1. The transmission is by mass action, so that $\beta(P)=\beta_{H} \Psi$. For all $T>0$, the system (21) has a unique nonnegative branch of periodic solutions, $\varphi_{T}$, that depend continuously on $T$.

2. There is no disease-associated death and the transmission is by standard incidence, so that $\beta(P)=\beta_{H} \frac{\Psi}{S+I+R}$ and $\gamma=0$. For $T>T_{*}$, system (21) has a unique nonnegative branch of periodic solutions, $\varphi_{T}$, that depend continuously on $T$, where $T_{*}$ is defined as in Lemma 6.

The system with autonomous spraying (22) has a periodic solution for every $\bar{\Theta}>0$. If $\bar{\Theta}$ is sufficiently small and the branch $\varphi_{T}$ is hyperbolic for $T$ sufficiently small (or, for Condition 2 , for $T \approx T_{*}$ ), there is a unique periodic solution. 
Corollary 12.1. Suppose the malaria transmission is modelled either by mass action or standard incidence. Then there exist $\epsilon_{1}, \ldots, \epsilon_{4}>0$ and $\Theta^{+}>$ 0 such that, for

$$
\gamma<\epsilon_{1}, \quad \delta<\epsilon_{2}, \quad h<\epsilon_{3}, \quad \alpha<\epsilon_{4}, \quad \bar{\Theta}<\Theta^{+},
$$

the following are true.

1. The system with spraying at fixed times (21) has a unique, nonnegative periodic solution that is asymptotically stable.

2. The system with incidence-based spraying (22) has a unique, nonnegative periodic solution that is orbitally asymptotically stable and enjoys the property of asymptotic phase.

\section{Discussion}

A mathematical model of malaria with insecticide effect has been proposed, where there is a mutant strain of mosquito that has complete immunity to the insecticide. The insecticide control is triggered when some critical number of new human malaria cases is detected. It was shown that this control strategy is asymptotically equivalent to spraying at periodic times, in that both systems share the same common endemic periodic orbits, and stability of the orbit in one system implies its stability in the other. A general version of this result is provided by Propositions 1-2.

The positive invariance of the nonnegative cone does not hold in general for this model, due to how the mutation is modelled. An implicit condition for the existence of a nonnegative, convex, positively invariant domain was provided by Theorem 1. An explicit condition is also available and is given by Theorem 4, assuming the mutation rate, $\epsilon$, is sufficiently small.

Following this, we consider the mosquito-only submodel. Theorem 3 outlines conditions under which, for small mutation rates, the mosquito submodel exhibits either a single mutant-only equilibrium, a mutant-only equilibrium and a single coexistence equilibrium, or a mutant-only equilibrium and a pair of coexistence equilibria; in all cases, the extinction equilibrium is also present. Numerous results pertaining to the persistence or development of bistability, when the mutation rate is small and positive, are presented in Theorems 6-7. 
The impulse effects are then re-introduced. Theorem 8 provides conditions under which the extinction equilibrium undergoes a saddle-node bifurcation. This bifurcation involves a biologically irrelevant periodic orbit and is not of great interest.

Theorem 9 demonstrates that, under certain conditions, the mutant-only equilibrium undergoes a saddle-node bifurcation. In this case, as the spraying efficacy $q$ increases and passes through the $q_{M}^{*}(\epsilon)$, a stable nonnegative coexistence equilibrium collides with the mutant-only equilibrium, becoming nonnegative and losing stability. The mutant-only equilbrium changes from a saddle to a sink.

The final explicit bifurcation result is Theorem 10. This theorem provides a somewhat complicated condition under which a codimension-two bifurcation can occur at the wild-type-only equilibrium, when $\epsilon=0$ and $q=q_{2}^{*}=1-\exp \left(-r_{w} T\left(1-\frac{K m}{\alpha_{m w} K w}\right)\right)$.

Numerical simulations were provided to reinforce the theoretical results on bistability of the coexistence periodic orbit and the mutant-only equilibrium. It was suggested that another bifurcation can occur when the spraying efficacy is significantly large. We conjecture that, under certain conditions, the stable coexistence periodic orbit collides with an unstable coexistence periodic orbit and both vanish, leaving room for the mutant-only equilibrium to become globally stable, when the spraying efficacy $q$ reaches some critical threshold.

From a policy perspective, this result is important. There is essentially a one-to-one correspondence between critical spraying thresholds and spraying periods (the defining equations for every critical spraying threshold can be inverted to solve for $T$ ), the latter of which are typically controllable. As such, another interpretation for the consequence of the above conjecture is that if spraying occurs too frequently, the mutant allele may become very prevalent in the mosquito population.

Finally, we returned to the human-mosquito dynamics by considering a simplified model in which all mosquitoes are assumed to be infectious. This simplification makes the model far more amenable to analysis. We are able to prove that, provided certain parameters are small, the correspondence between spraying at fixed times and spraying according to the autonomous tracking model is even more strict. Specifically, the period $T$ and infection threshold $\bar{\Theta}$ uniquely determine each other, provided each is sufficiently small. These results hold for mass-action or standard-incidence infection rates and are summarized in Theorem 11-12 and Corollary 12.1. 
In conclusion, the mathematical model (5) and its various submodels exhibit a wealth of different qualitative dynamics, including bistability, several bifurcations of fixed points and periodic orbits. We have shown that spraying at fixed times is asymptotically equivalent to spraying when a critical number of new human infections are detected. As such, one strategy may be more or less costly to implement yet yield the same long-term result as the other. A sufficiently powerful insecticide, sprayed too frequently, could result in the mutant allele becoming very common in the mosquito population, thereby reducing its efficacy as a control method. Care must therefore be taken to not spray too often. How frequently to spray to avoid this problem can be informed using the results from Section 3, although the picture is incomplete, as discussed in this final section, as well as in Section 4. Finally, by appealing to a simpler model, we see that whether spraying at fixed times or according to an infection threshold, there can be only one endemic periodic orbit, provided the period or threshold is small enough.

\section{Acknowledgements}

KEC is supported by an Ontario Graduate Scholarship. RJS? is supported by an NSERC Discovery Grant. For citation purposes, please note that the question mark in "Smith?" is part of his name.

\section{References}

[1] Agur Z.; Cojocaru, L.; Mazor, G.; Anderson, R.; Danon, Y. Pulse mass measles vaccination across age cohorts. Proc. Natl. Acad. Sci. USA 1993, 90, 11698-11702.

[2] M. Al-arydah and R.J. Smith? (2011) Controlling malaria with Indoor Residual Spraying in Spatially Heterogeneous Environments, Mathematical Biosciences and Engineering 8(4), 880-914.

[3] D.D. Bainov and P.S. Simeonov, Systems with Impulsive Effect; Ellis Horwood Ltd.: Chichester, UK, 1989. 
[4] D.D. Bainov and P.S. Simeonov, Impulsive Differential Equations: Periodic Solutions and Applications, Longman Scientific \& Technical, Burnt Mill [1993].

[5] Bainov, D.D.; Simeonov, P.S. Impulsive Differential Equations: Asymptotic Properties of the Solutions; World Scientific: Singapore, 1995.

[6] J. G. Breman, The ears of the hippopotamus: manifestations, determinants, and estimates of the malaria burden, The American Journal of Tropical Medicine and Hygiene, vol. 64, no. 1-2, supplement, pp. 1-11, 2001.

[7] M. Finkel Malaria: Stopping a global killer, National Geographic, July 2007.

[8] H. Flanders, Differentiation Under the Integral Sign, The American Mathematical Monthly, Vol. 80, No. 6, 615-627 [1973].

[9] J. Hemingway, The role of vector control in stopping the transmission of malaria: threats and opportunities, Philosophical Transactions of the Royal Society B 369 (2014): 20130431.

[10] K.E. Jones, N.G. patel, M.A. Levy, A. Storeygard, D. Balk, J.L. Gittleman and P. Daszak, Global trends in emerging infectious diseases Nature 451 (2008), 990-993.

[11] J. Kato et. al, Bounded Solutions and Periodic Solutions to Linear Differential Equations in Banach Spaces, Vietnam Journal of Mathematics 30: SI 561-575 [2002].

[12] Lakshmikantham, V.; Bainov, D.D.; Simeonov, P.S. Theory of Impulsive Differential Equations; World Scientific: Singapore, 1989.

[13] A. D. Lopez, C. D. Mathers, M. Ezzati, D. T. Jamison and C. J. Murray, Global and regional burden of disease and risk factors, 2001: Systematic analysis of population health data, Lancet, 367 (2006), 1747-1757.

[14] M. L. Mabaso, B. Sharp and C. Lengeler, Historical review of malarial control in southern Africa with emphasis on the use of indoor residual house-spraying, Trop. Med. Int. Health, 9 (2004), 846-856. 
[15] K. Macintyre, J. Keating, Y. B. Okbaldt, M. Zerom, S. Sosler, T. Ghebremeskel and T. P. Eisele, Rolling out insecticide treated nets in Eritrea: Examining the determinants of possession and use in malarious zones during the rainy season, Trop. Med. Int. Health, 11 (2006), 824833.

[16] Miron, R.E.; Smith?, R.J. Modelling imperfect adherence to HIV induction therapy. BMC Infect. Dis. 2010, 10, doi:10.1186/1471-2334-10-6.

[17] F. Nosten and R. N. Price, New antimalarials: a risk-benefit analysis, Drug Safety, vol. 12, no. 4, pp. 264-273, 1995.

[18] P. van de Perre and J.-P. Dedet, Vaccine effcacy: winning a battle (not war) against malaria, The Lancet, vol. 364, no. 9443, pp. 1380-1383, 2004 .

[19] Roberts, M.G.; Kao, R.R. The dynamics of an infectious disease in a population with birth pulses. Math. Biosci. 1998, 149, 23-36.

[20] B.L. Sharp, F.C. Ridl, D. Govender, J. Kuklinski and I. Kleinschmidt Malaria vector control by indoor residual insecticide spraying on the tropical island of Bioko, Equatorial Guinea Malaria Journal 6 (2007): 52.

[21] K. D. Silué, G. Raso, A. Yapi, P. Vounatsou, M. Tanner, E. K. N'Goran and J. Utzinger, Spatially-explicit risk profiling of Plasmodium falciparum infections at a small scale: A geo- statistical modelling approach, Malar J., 7 (2008), 111.

[22] R.J. Smith? and S.D. Hove-Musekwa, Determining Effective Spraying Periods to Control Malaria via Indoor Residual Spraying in sub-Saharan Africa, Journal of Applied Mathematics and Decision Sciences Vol. 2008, Article ID 745463 [2008].

[23] R. W. Snow, C. A. Guerra, A. M. Noor, H. Y. Myint and S. I. Hay, The global distribution of clinical episodes of Plasmodium falciparum malaria, Nature, 434 (2005), 214-217.

[24] Stone L.; Olinky, R.; Huppert, A. Seasonal dynamics of recurrent epidemics. Nature 2007, 446, 533-536. 
[25] P. I. Trigg and A. V. Kondrachine, Commentary: Malaria control in the 1990s, Bull. World Health Organ., 76 (1998), 11-16. surveillance

[26] World health Organization, Indoor residual spraying: An operational manual for IRS for malaria transmission, control and elimination. Second edition (2015) http://www.who.int/malaria/publications/ atoz/9789241508940/en/

[27] World Health Organisation, Malaria factsheet Number 94 http://www.who.int/mediacentre/factsheets/fs094/en/

\section{Appendix: Proofs}

\section{Proof of Theorem 1}

The impulse effect clearly maps $\bar{\Omega}$ into itself. Second, since $\bar{\Omega}$ is compact, $\xi \equiv$ $\sup _{P \in \bar{\Omega}} \beta(P)$ exists. But then, for all $(P, \Theta) \in \bar{\Omega}$, we have $\Theta^{\prime} \leq \eta \xi \pi / \mu_{H} \equiv C$, so that if an impulse effect occurs at some time, $t_{k}$, then the next impulse effect can occur only as early as time $t_{k}+\bar{\Theta} / C$. Consequently, there is a finite amount of time between each impulse effect, so that no solution can have an accumulation point on the hypersurface $\Theta=\bar{\Theta}$. We may therefore conclude that if $\Omega_{b_{w}}^{*}$ is positively invariant, then solutions are defined for all (positive) time [4].

To see the invariance, note that it is only necessary to examine the invariance of the $P$ component of (5). If $P_{0}$ is a point on the boundary of the nonnegative cone, it is clear that, provided the state-dependent coefficient $b_{w}$ is nonnegative at $P_{0}$, the forward flow from $P_{0}$ will remain nonnegative for some small positive amount of time. It is thus sufficient to determine a domain on which $b_{w}$ remains nonnegative for all time. Since $b_{w}=b_{w}^{0}-K_{b w w} V_{w}-K_{b w m} V_{m}$, where $V_{w}=M_{w}+N_{w}$ and $V_{m}=M_{m}+N_{m}$, the nonnegativity of $b_{w}$ is determined by the dynamics of system (6). Specifically, the condition $b_{w} \geq 0$ is equivalent to the positive invariance of $\Omega_{b_{w}}$ under the flow of (6). The condition $b_{w} \geq 0$ accounts for the restriction on $M_{w}, M_{m}, N_{w}$ and $N_{m}$ in the expression for $\Omega_{b_{w}}^{*}$. Finally, the differential inequality $(S+I+R)^{\prime} \leq \pi-\mu_{H}(S+I+R)$ accounts for the other restriction, $0 \leq S+I+R \leq \frac{\pi}{\mu_{H}}$, present in $\Omega_{b_{w}}^{*}$. Uniqueness is guaranteed by standard results [4]. 


\section{Proof of Proposition 1}

Let (8) have a unique $T$-periodic solution, $\varphi(t)$, and let (10) hold. Consider the point $\left(\varphi\left(0^{+}\right), 0\right) \equiv z_{0}^{+} \in \Omega \times \mathbb{R}_{+} \equiv X$. The left limit of the forward flow $\phi: X \times \mathbb{R}_{+} \rightarrow X$ of (9) at time $T$ is

$$
\begin{aligned}
\phi\left(z_{0}^{+}, T^{-}\right)=\lim _{t \rightarrow T^{-}} \phi\left(z_{0}^{+}, t\right) & =\left(\varphi\left(T^{-}\right), \int_{0}^{T} u(\varphi(t)) d t\right) \\
& =\left(\varphi\left(T^{-}\right), \bar{\theta}\right) .
\end{aligned}
$$

Hence an impulse occurs along the forward orbit from $z_{0}^{+}$at time $t=T$. By periodicity of $\varphi(t)$ and the impulse condition, we have

$$
\phi\left(z_{0}^{+}, T^{+}\right)=\lim _{t \rightarrow T^{+}} \phi\left(z_{0}^{+}, t\right)=(\varphi(0), 0)=z_{0}^{+} .
$$

Note also that $\bar{\theta} \neq 0$, since $u$ is strictly positive along $\varphi$. It follows that there is always a finite amount of time between impulses. We may therefore conclude that equation (9) has a $T$-periodic solution. Moreover, it is unique up to phase shift. If (9) had another $T$-periodic solution, say $\left(x_{1}(t), \theta_{1}(t)\right)$, which had an impulse effect at $t=T$, then $x_{1}(t+T)$ would be a $T$-periodic solution of (8). By uniqueness of periodic solutions of the fixed-time equation, we would then have $x_{1}(t+T)=\varphi(t)$. By the impulse condition, we have $\tilde{\theta}\left(0^{+}\right)=0=\theta_{1}\left(T^{+}\right)$. Finally, for $t \in(0, T)$,

$$
\begin{aligned}
\theta(t) & =\theta\left(0^{+}\right)+\int_{0}^{t} u(\varphi(s)) d s \\
& =\theta_{1}\left(T^{+}\right)+\int_{0}^{t} u\left(x_{1}(T+s)\right) d s \\
& =\theta_{1}\left(T^{+}\right)+\int_{T}^{T+t} u\left(x_{1}(s)\right) d s \\
& =\theta_{1}(T+t) .
\end{aligned}
$$

Therefore $\left(x_{1}, \theta_{1}\right)$ is a phase shift of $(\varphi, \tilde{\theta})$. There can thus be only one $T$-periodic solution of the autonomous equation (9) up to phase shift equivalence. The converse statement is obvious.

\section{Proof of Proposition 2}

Without loss of generality, we have $\tilde{\varphi}=(\varphi, \theta)$ for a $T$-periodic function $\theta$ satisfying $\theta\left(0^{+}\right)=0$. The variational equation associated to the periodic 
orbit $\varphi(t)$ is

$$
\begin{aligned}
\dot{z} & =\frac{d g}{d x}(\varphi(t)) z, & & \neq k T, \\
\Delta z & =\frac{d a}{d x} z, & t & =k T .
\end{aligned}
$$

Conversely, the variational equation at $\tilde{\varphi}(t)$ is

$$
\begin{aligned}
& \dot{w}=\left[\begin{array}{c|c}
\frac{d g}{d x}(\varphi(t)) & \vdots \\
& 0
\end{array}\right] w, \quad t \neq k T \\
& \Delta w=\left[\begin{array}{ccc|c} 
& & d a & \\
& \frac{d a}{d x} & & \xi \\
& & & \\
\hline 0 & \cdots & 0 & -1
\end{array}\right] w, \quad t=k T,
\end{aligned}
$$

where

$$
\xi=g\left(\varphi\left(T^{+}\right)\right)-g(\varphi(T))-\frac{d a}{d x} g(\varphi(T)),
$$

and $w=\left(w_{1: n}, w_{n+1}\right)$. It follows by classical results [4] that the above equation (24) has a nontrivial $T$-periodic solution, namely $\tilde{\varphi}^{\prime}(t)$. Without loss of generality, we may assume $\tilde{\varphi}^{\prime}(0)=e^{n+1}$. If $\varphi(t)$ is exponentially stable, then there exist $n$ linearly independent solutions $v_{1}, \ldots, v_{n}$ of (23) satisfying $v_{j}(0)=e^{j}$ for $j=1, \ldots, n$. It is then easy to check that $\left(v_{j}, 0\right)$ are linearly independent solutions of (24). Therefore a monodromy matrix for the linearized tracking system is

$$
M=\left[\begin{array}{cccc}
v_{1}(T) & \cdots & v_{n}(T) & \varphi^{\prime}(T) \\
0 & \cdots & 0 & \theta^{\prime}(T)
\end{array}\right]
$$

The matrix $M$ has a block structure; the floquet multipliers are precisely $\theta^{\prime}(T)=\theta^{\prime}(0)=1$ and the multipliers of $\varphi(t)$, which are, by hypothesis, within the unit disc, since this solution is exponentially stable. Therefore $\tilde{\varphi}(t)$ is orbitally asymptotically stable and has the property of asymptotic phase [4]. The converse follows by similar reasoning. 


\section{Proof of Lemma 1}

The $V_{m}$ nullcline is the solution of the second-degree equation

$$
\begin{aligned}
\mathcal{N}\left(V_{w}, V_{m}\right)= & V_{m}^{2}\left(\frac{r_{m}}{K_{m}}\right)+V_{m} V_{w}\left(\frac{r_{m} \alpha_{m w}}{K_{m}}+\epsilon K_{b w m}\right)+V_{w}^{2}\left(\epsilon K_{b w w}\right) \\
& +V_{m}\left(-r_{m}\right)+V_{w}\left(-\epsilon b_{w}^{0}\right)=0 .
\end{aligned}
$$

Its determinant, which we consider as a function of $\epsilon$, is

$$
\begin{aligned}
\operatorname{det}(\epsilon) & =\frac{1}{4}\left[\left(\frac{r_{m} \alpha_{m w}}{K_{m}}+\epsilon K_{b w m}\right) r_{m} \epsilon b_{w}^{0}-\epsilon K_{b w m} r_{m}^{2}-\frac{r_{m}}{K_{m}} \epsilon^{2}\left(b_{w}^{0}\right)^{2}\right] \\
& =\frac{\epsilon r_{m}}{4}\left[r_{m}\left(\frac{b_{w}^{0} \alpha_{m w}}{K_{m}}-K_{b w w}\right)-\epsilon b_{w}^{0}\left(\frac{b_{w}^{0}}{K_{m}}-K_{b w m}\right)\right] .
\end{aligned}
$$

Thus, the determinant vanishes if $\epsilon=0$ or if $\epsilon=\hat{\epsilon}$, where

$$
\hat{\epsilon} \equiv \frac{r_{m}\left(\frac{b_{w}^{0} \alpha_{m w}}{K_{m}}-K_{b w m}\right)}{b_{w}^{0}\left(\frac{b_{w}^{0}}{K_{m}}-K_{b w w}\right)} .
$$

By the hypothesis on the equalities (13), $\hat{\epsilon}$ is either well-defined and nonzero, zero, or is undefined. If $\hat{\epsilon}$ is undefined, we will formally write $\hat{\epsilon}=\infty$. The case $\hat{\epsilon}=\infty$ occurs when $\frac{b_{w}^{0}}{K_{m}}=K_{b w m}$, so that the determinant reduces to

$$
\operatorname{det}(\epsilon)=\frac{\epsilon r_{m}^{2}}{4}\left(\frac{b_{w}^{0} \alpha_{m w}}{K_{m}}-K_{b w w}\right) .
$$

The determinant is nonzero when $\epsilon \neq 0$. In summary, if we formally write

$$
\epsilon_{1}=\left\{\begin{array}{cc}
\hat{\epsilon}, & \hat{\epsilon} \neq 0, \\
\infty, & \hat{\epsilon}=0,
\end{array}\right.
$$

then $\operatorname{det}(\epsilon) \neq 0$ provided $0<\epsilon<\epsilon_{1}$.

Next, the discriminant of $(25)$ is

$$
\begin{aligned}
\Delta(\epsilon) & =\left(\frac{r_{m} \alpha_{m w}}{K_{m}}+\epsilon K_{b w m}\right)^{2}-\frac{4 r_{m} \epsilon K_{b w w}}{K_{m}} \\
& =\left(\frac{r_{m} \alpha_{m w}}{K_{m}}\right)^{2}+\epsilon\left(\frac{2 r_{m}}{K_{m}}\left(\alpha_{m w} K_{b w m}-2 K_{b w w}\right)+\epsilon K_{b w m}^{2}\right) .
\end{aligned}
$$

Since $\Delta(\epsilon)$ is continuous and $\Delta(0)>0$, it follows that there exists $\epsilon_{2}>0$ such that if $0<\epsilon<\epsilon_{2}$, we have $\Delta>0$. If we define $\epsilon_{0}=\min \left\{\epsilon_{1}, \epsilon_{2}\right\}$, then we have $\Delta>0$ and det $\neq 0$ whenever $0<\epsilon<\epsilon_{0}$. This indicates that (25) describes a non-degenerate hyperbola. 


\section{Proof of Lemma 2}

Let $0<\epsilon<\epsilon_{0}$, so that the $V_{m}$ nullcline is a hyperbola. Notice that $(0,0)$, $\left(0, K_{m}\right)$ and $\left(b_{w}^{0} / K_{b w w}, 0\right)$ are all solutions of the equation $\mathcal{N}\left(V_{w}, V_{m}\right)=0$, and the first two constitute the only solutions of the equation $\mathcal{N}\left(0, V_{m}\right)=0$. Implicitly differentiating both sides $\mathcal{N}\left(V_{w}, V_{m}\right)=0$ with respect to $V_{m}$ at $(0,0)$, we obtain the equation $r_{m}+\epsilon b_{w}^{0} V_{w}^{\prime}(0,0)=0$, which gives

$$
V_{w}^{\prime}(0,0)=-\frac{r_{m}}{\epsilon b_{w}^{0}}<0
$$

Implicitly differentiating with respect to $V_{m}$ at $\left(b_{w}^{0} / K_{b w w}, 0\right)$ results in

$$
V_{w}^{\prime}\left(b_{w}^{0} / K_{b w w}, 0\right)=\frac{r_{m}}{\epsilon b_{w}^{0}}\left(1-\frac{\alpha_{m w} b_{w}^{0}}{K_{m} K_{b w w}}\right)-\frac{K_{b w m}}{K_{b w w}}<0
$$

where the inequality follows by the assumption in equation (14). Finally, implicitly differentiating at $\left(0, K_{m}\right)$ gives

$$
V_{w}^{\prime}\left(0, K_{m}\right)=\frac{r_{m}}{-r_{m} \alpha_{m w}+\epsilon\left(b_{w}^{0}-K_{m} K_{b w m}\right)}<0
$$

provided $\epsilon$ is sufficiently small; say, $\epsilon<\epsilon^{0}$. Inequalities (26) and (28) indicate that the points $(0,0)$ and $\left(0, K_{m}\right)$ must lie on distinct branches of the hyperbola; otherwise, there would need to be a branch with two critical points. Similarly, inequalities (26) and (27) guarantee that the points $(0,0)$ and $\left(b_{w}^{0} / K_{b w w}, 0\right)$ must lie on different branches. We conclude that $\left(b_{w}^{0} / K_{b w w}, 0\right)$ and $\left(0, K_{m}\right)$ lie on the same branch of the hyperbola, while $(0,0)$ is on the other. From the above analysis, we can also conclude that the portion of the first branch contained in the nonnegative quadrant can be identified with the graph of a strictly decreasing function $M:\left[0, K_{m}\right] \rightarrow\left[0, b_{w}^{0} / K_{b w w}\right]$.

\section{Proof of Lemma 4}

Assume $\epsilon>0$ is sufficiently small to guarantee the conditions of Theorem 3 are satisfied. Let $W\left(V_{m}\right)$ and $M\left(V_{m}\right)$ denote, respectively, the $V_{w}$ and $V_{m}$ nullclines (15) and (25) in the nonnegative quadrant. Let $V_{w}^{0}$ and $V_{m}^{0}$ denote, respectively, the intersections of $W$ and $M$ with the line $V_{w}=0$. A quick 
calculation reveals

$$
\begin{aligned}
W^{0} & =\frac{K_{w}\left(r_{w}-\epsilon b_{w}^{0}\right)}{\alpha_{w m} r_{w}-\epsilon K_{b w m} K_{w}}, & M^{0} & =K_{m}, \\
W(0) & =\frac{K_{w}\left(r_{w}-\epsilon b_{w}^{0}\right)}{r_{w}-\epsilon K_{b w w} K_{w}}, & M(0) & =\frac{b_{w}^{0}}{K_{b w w}} .
\end{aligned}
$$

We have

$$
V_{w}^{\prime}(M(0), 0)=\frac{b_{w}^{0}}{K_{b w w}}\left(1-\frac{b_{w}^{0}}{K_{b w w} K_{w}}\right)<0,
$$

by the hypothesis $b_{w}^{0}>K_{b w w} K_{w}$. Also

$$
\frac{\partial V_{m}^{\prime}}{\partial V_{w}}(M(0), 0)=-\epsilon b_{w}^{0}<0 .
$$

By inequalities (29)-(30), if $\gamma$ is given a parameterization $\gamma:[0, \infty) \rightarrow \mathbb{R}_{+}^{2}$ such that $\gamma(0)=\left(b_{w}^{0} / K_{b w w}, 0\right)$, then both components of $\gamma$ are initially increasing. The condition $b_{w}^{0}>K_{b w w} K_{w}$ implies that $W(0)$ is a decreasing function of $\epsilon$, so that if $0<\epsilon \ll \frac{r_{w}}{K_{b w w} K_{w}}$, we will have $0<W(0)<M(0)$. Consequently, there are no nullclines with $V_{w}$ coordinate greater than $b_{w}^{0} / K_{b w w}$ in the nonnegative quadrant. We conclude that $\gamma$ is increasing for all time. The inequality (30) implies that $V_{m}^{\prime}>0$ along the line connecting $\gamma(0)$ to the origin (except at $\gamma(0)$, where $V_{m}^{\prime}=0$ ). Finally, the line $V_{w}=0$ is a $V_{w}$ nullcline, so is itself positively invariant since $\frac{\partial V_{m}^{\prime}}{\partial V_{m}}>0$ at the origin. It follows that $\Omega$ is positively invariant. See Figure 4 for a visualization.

That $\Omega$ is the largest nonnegative positively invariant set can be explained as follows. Any trajectory that is initially positive but not contained in $\Omega$ must satisfy $V_{m}^{\prime}<0$ and $V_{w}^{\prime}<0$ until it traverses a nullcline. However, since $\Omega$ contains all portions of all nullclines contained in the nonnegative quadrant, and the "upper" boundary of $\Omega$ is a solution curve, the proposed trajectory cannot cross it. Since the upper boundary of $\Omega$ contains no equilibrium point, the proposed trajectory cannot have a limit point on the upper boundary of $\Omega$. Therefore the trajectory must eventually leave the nonnegative quadrant.

\section{Proof of Theorem 4}

Verification that inequalities (16) imply the hyperbolic criteria is straightforward. Next, as in the proof of Lemma 4, the first condition of (16) guarantees that $W(0)$ is a decreasing function of $\epsilon$ and, when $\epsilon$ is small, that 
$W(0)<M(0)$. The second inequality of (16) is equivalent to

$$
\max \left\{M^{0}, W^{0}\right\}<\frac{b_{w}^{0}}{K_{b w m}}
$$

when $\epsilon=0$. Since $M^{0}$ is constant and $W^{0}$ is continuous with respect to $\epsilon$, inequlity (31) persists for $\epsilon>0$ small.

Consider the region defined by the inequality $b_{w} \geq 0$, contained in $\mathbb{R}_{+}^{2}$. This is the region defined by

$$
V_{w} \leq \frac{b_{w}^{0}}{K_{b w w}}-\frac{K_{b w m}}{K_{b w w}} V_{m}
$$

The $V_{w}$ intercept is $\frac{b_{w}^{0}}{K_{b w w}}=M(0)$, while the $V_{m}$ intercept is the right-hand side of (31). We conclude from the above analysis that the graph of the boundary of (32) lies above the the graph of both $W$ and $M$, in the nonnegative quadrant. Consequently, the signs of $\dot{V}_{m}$ and $\dot{V}_{w}$ are constant above both the graph of $\max \{W, M\}$ and below the graph of the boundary of (32). Both of these derivatives are nonpositive in this region, since $\dot{V}_{w}(M(0), 0)<0$ by (29) and $\dot{V}_{m}\left(0, b_{w}^{0} / K_{b w m}\right)<\dot{V}_{m}\left(0, M^{0}\right)=0$. This allows us to conclude that (32) is positively invariant, since this set is a strict subset of the positively invariant set $\Omega$ from Theorem 4 , and the boundaries of (32) consist of boundaries of $\Omega$, together with the line $V_{w}=\frac{b_{w}^{0}}{K_{b w w}}-\frac{K_{b w m}}{K_{b w w}} V_{m}$, on which both $\dot{V}_{w}$ and $\dot{V}_{m}$ are nonpositive (in fact negative, except $\dot{V}_{m}(M(0), 0)$, which has been discussed in Theorem 4). See Figure 4 for a visualization. The result follows if one recalls that $\Omega_{b_{w}}$ of Theorem 1 is defined by inequality (32).

\section{Proof of Proposition 3}

Since the only biologically relevant equilibrium points lie on the the line $V_{w}=0$, which is a $V_{w}$ nullcline, it follows that there are no periodic orbits. The extinction equilibrium's eigenvalues are $\lambda_{1}^{0}=r_{w}-\epsilon b_{w}^{0}$ and $\lambda_{2}^{0}=r_{m}$, so that $(0,0)$ is unstable for all $\epsilon \in \mathbb{R}$; in particular, when $\epsilon$ is small, both its eigenvalues are positive. Consequently, there are no heteroclinic circuits between the equilibria. By the Poincaré-Bendixson theorem, it follows that the mutant-only equilibrium, $\left(0, K_{m}\right)$, is globally attracting on $\Omega \backslash\{(0,0)\}$. 


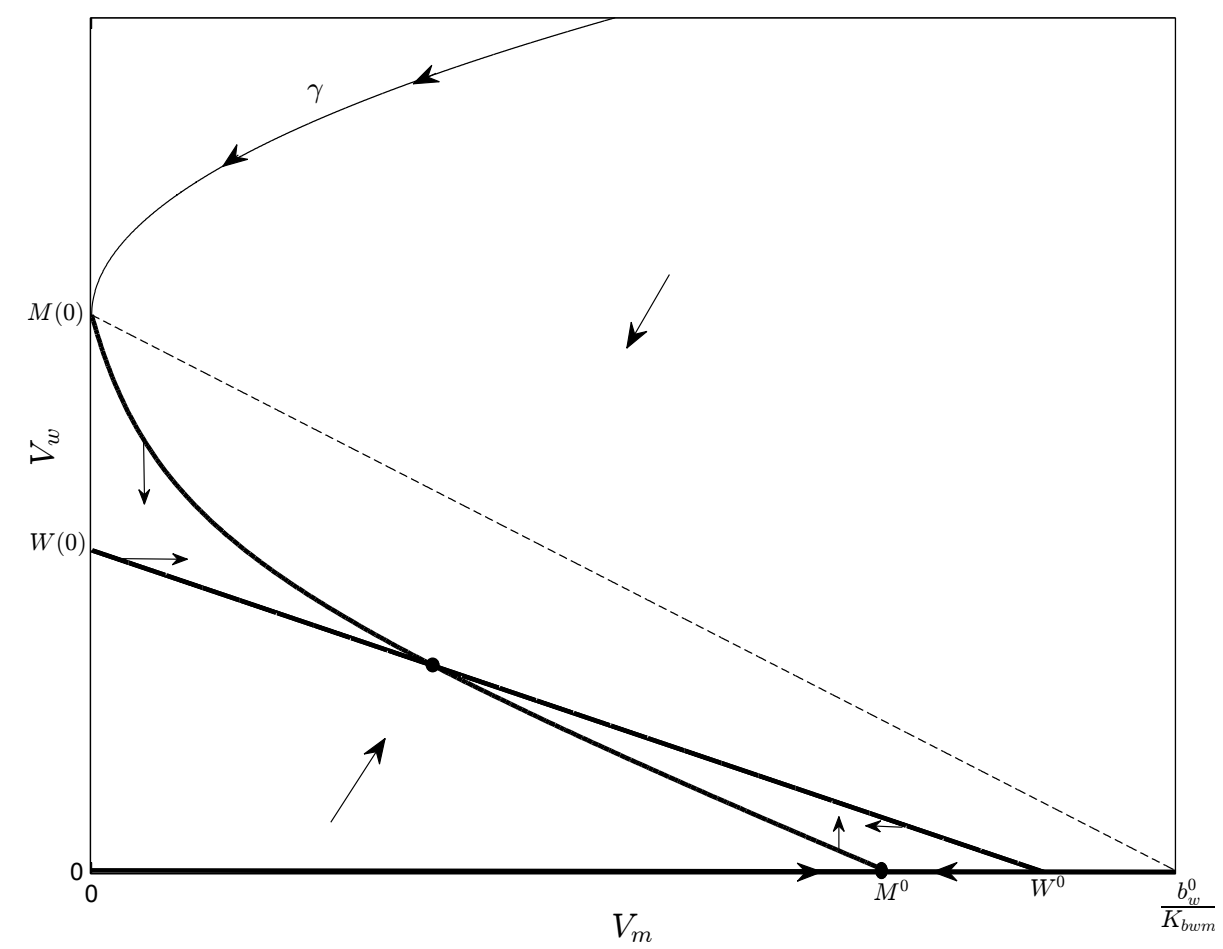

Figure 4: A typical phase portrait of the system (12). In this case, there is a single coexistence equilibrium that appears to be asymptotically stable. Nullclines are indicated by bold lines. The backward orbit from $(M(0), 0)$, denoted $\gamma$ (see Lemma 4), is present in the figure. The dashed line indicates the "upper" boundary of the region (32) of Theorem 4. Equilibrium points are indicated by dots, and arrow directions indicate approximate directions of solution velocity in forward time. 


\section{Proof of Theorem 5}

Let $W\left(V_{m}\right)$ and $M\left(V_{m}\right)$ denote, respectively, the $V_{w}$ and $V_{m}$ nullclines (15) and (25) in the nonnegative quadrant. Let $W^{0}$ and $M^{0}$ denote, respectively, the intersections of $W$ and $M$ with the line $V_{w}=0$. We have

$$
\begin{aligned}
W^{0} & =\frac{K_{w}\left(r_{w}-\epsilon b_{w}^{0}\right)}{\alpha_{w m} r_{w}-\epsilon K_{b w m} K_{w}}, & M^{0} & =K_{m}, \\
W(0) & =\frac{K_{w}\left(r_{w}-\epsilon b_{w}^{0}\right)}{r_{w}-\epsilon K_{b w w} K_{w}}, & M(0) & =\frac{b_{w}^{0}}{K_{b w w}} .
\end{aligned}
$$

The condition $K_{m}<\alpha_{m w} K_{w}$ implies that $W$ and $M$, when $\epsilon=0$, cannot intersect on the interior of $\mathbb{R}_{+}^{2}$ (see the proof of Theorem 6 for the explicit intersection point), so that there can be at most three equilibrium points, all of which must be on the boundary. This fact holds true for $\epsilon>0$ sufficiently small, by continuity of $W$ and $M$ with respect to $\epsilon$.

The condition $b_{w}^{0}>K_{b w w} K_{w}$ implies that $W(0)$ is a decreasing function of $\epsilon$, so that if $0<\epsilon \ll \frac{r_{w}}{K_{b w w} K_{w}}$, we will have $0<W(0)<M(0)$. But then, due to the hyperbolic criteria, Lemma 2 implies that if there is a single coexistence equilibrium at which the nullclines (15) and (25) intersect, then we must have $M^{0} \leq W^{0}$. One will notice that, when $\epsilon=0$, the inequality $M^{0}<W^{0}$ is equivalent to condition A1, but since $M^{0}$ and $W^{0}$ are continuous functions of $\epsilon$ at $\epsilon=0$, the inequality must persist for $\epsilon$ small. On the other hand, when $\epsilon=0$, the condition $M_{0}=W_{0}$ is equivalent to the first equality of condition A2. The other two inequalities of condition A2 are necessary for the condition $M^{0} \leq W^{0}$ to persist when $\epsilon>0$ is small. This establishes necessity.

If condition A1 holds, so that we have $M^{0}<W^{0}$ and $W(0)<M(0)$ for $\epsilon>0$ small, the intermediate value theorem guarantees that the nullclines must intersect at least once, and there can be no more than one intersection in the positive quadrant because $M$ is convex there. Conversely, if condition A2 holds, then $W^{0} \leq M^{0}$ for $\epsilon>0$ small, and one will find that the third inequality of condition A2 implies that $W^{\prime}\left(M^{0}\right)<M^{\prime}\left(M^{0}\right)$, provided $\epsilon>0$ is small, so that for $0 \ll v<V_{m}$, we have $M(v)<W(v)$. Again, since $W(0)<M(0)$, the intermediate value theorem guarantees that $W$ and $M$ intersect in the interval $(0, v)$, and there can be only one intersection in the positive quadrant, due to the convexity of $M$. We have proven sufficiency of conditions A1 and A2. 
Next we discuss attractivity. It has already been demonstrated in Proposition 3 that the extinction equilibrium is unstable. If both it and the mutantonly equilibrium are unstable, then, by similar reasoning as that of the proof of Proposition $3, E_{0}$ will either be globally attracting, or there will be a stable periodic orbit that encloses it. We will now rule out the existence of periodic orbits.

Suppose conditions A1 or A2 of the theorem hold. We have

$$
V_{w}^{\prime}(M(0), 0)=\frac{b_{w}^{0}}{K_{b w w}}\left(1-\frac{b_{w}^{0}}{K_{b w w} K_{w}}\right)<0,
$$

by the hypothesis $b_{w}^{0}>K_{b w w} K_{w}$. Also,

$$
V_{m}^{\prime}(W(0), 0)=\epsilon W(0)\left(b_{w}^{0}-K_{b w w} W(0)\right)>0,
$$

provided $b_{w}^{0}>K_{b w w} W(0)>0$. That is, to maintain the above inequality, we require

$$
b_{w}^{0}>K_{b w w} K_{w}\left(\frac{r_{w}-\epsilon b_{w}^{0}}{r_{w}-\epsilon K_{b w w} K_{w}}\right)>0 .
$$

When $\epsilon=0$, the inequality holds due to the hypothesis $b_{w}^{0}>K_{b w w} K_{w}$. By continuity, (33) holds for $\epsilon$ sufficiently small. Finally, we have

$$
\frac{\partial V_{m}^{\prime}}{\partial V_{w}}(M(0), 0)=-\epsilon b_{w}^{0}<0 .
$$

Therefore it follows that, when $\epsilon$ is sufficiently small, the region

$$
\Omega=\left\{\left(V_{w}, V_{m}\right) \in \mathbb{R}_{2}^{+}: 0 \leq V_{m} \leq E_{0}^{1}, W\left(V_{m}, \epsilon\right) \leq V_{w} \leq M\left(V_{m}\right)\right\}
$$

is postively invariant. However, any periodic orbit enclosing $E_{0}$ must intersect $\Omega$. Hence there can be no periodic orbit.

As for the sufficient condition for instability of the mutant-only equilibrium, we observe that one of the eigenvalues of its linearization is

$$
r_{w}\left(1-\frac{\alpha_{w m} K_{m}}{K_{w}}\right)-\epsilon\left(b_{w}^{0}-K_{b w m} K_{m}\right),
$$

so that the equilibrium will be unstable if the above is positive. When conditon A1 holds, the above is positive when $\epsilon=0$, as stated in the theorem. 


\section{Proof of Theorem 6}

Part 1: Existence of equilibria and their stability. When $\epsilon=0$ and the inequalities (17) are satisfied, there are four equilibria: the extinction equilibrium, $\overline{0}=(0,0)$; the wild-type-only equilibrium, $W_{0}=\left(K_{w}, 0\right)$; the mutantonly equilibrium, $M_{0}=\left(0, K_{m}\right)$; and a coexistence equilibrium,

$$
E_{0}=\left(\frac{K_{w}-\alpha_{w m} K_{m}}{1-\alpha_{w m} \alpha_{m} w}, \frac{K_{m}-\alpha_{m w} K_{w}}{1-\alpha_{w m} \alpha_{m w}}\right)
$$

The linearizations, $L x$, at these equilibria satisfy the following:

$$
\begin{aligned}
\sigma(L \overline{0}) & =\left\{r_{w}, r_{m}\right\}, & \operatorname{det}\left(L E_{0}\right) & =\frac{r_{w} r_{m} E_{0}^{1} E_{0}^{2}}{K_{w} K_{m}}\left(1-\alpha_{w m} \alpha_{m w}\right), \\
\sigma\left(L W_{0}\right) & =\left\{-r_{w}, r_{m}\left(1-\frac{\alpha_{m w} K_{w}}{K_{m}}\right)\right\}, & \sigma\left(L M_{0}\right) & =\left\{r_{w}\left(1-\frac{\alpha_{w m} K_{m}}{K_{w}}\right),-r_{m}\right\} .
\end{aligned}
$$

From the above, we conclude that $\overline{0}$ is a source, $W_{0}$ and $M_{0}$ are sinks, $E_{0}$ is a saddle and their linearizations are invertible. By the implicit function theorem, these equilibria persist for $\epsilon>0$ small, and they have the same stability. The extinction and mutant-only equilibria do not depend on $\epsilon$, and the coexistence equilibrium is strictly positive, which means that these equilibria will all remain nonnegative when $\epsilon$ is small. Also, we note that

$$
L W_{0}=\left[\begin{array}{cc}
r_{m}\left(1-\frac{\alpha_{m w} K_{w}}{K_{m}}\right) & \cdots \\
0 & -r_{w}
\end{array}\right]
$$

which implies that the implicit function $W_{0}(\epsilon)=(W(\epsilon), M(\epsilon))$ describing the perturbation of the wild-type-only equilibrium satisfies, by the implicit function theorem,

$$
M^{\prime}(0)=\frac{-r_{w} K_{w}\left(b_{w}^{0}-K_{b w w} K_{w}\right)}{r_{w} r_{m}\left(1-\frac{\alpha_{m w} K_{w}}{K_{m}}\right)}>0,
$$

where positivity is guaranteed by inequalities (17). It follows that $W_{0}(\epsilon)$ is nonnegative for $\epsilon>0$ small. In conclusion, all equilibria described in the theorem exist and their stability is as stated.

Part 2: Nonexistence of periodic orbits. An argument similar to the proof 
of Theorem 5 can be used to show that no periodic orbit can enclose $W_{0}(\epsilon)$. Since $\overline{0}$ and $M_{0}(\epsilon)$ both lie on the invariant $W$ nullcline, $V_{w}=0$, neither of these can be enclosed within a periodic orbit. From the linearizations in Part 1 , all equilibria are hyperbolic. Since the only other equilibrium $\left(E_{0}\right)$ is a saddle, by index theory, we conclude that there are no periodic orbits.

Part 3: Existence of the heteroclinic orbit. To exhibit the heteroclinic orbit, we consider the $\alpha$-limit set of a point, $x_{0}$, on the stable manifold of the saddle-type coexistence equilibrium, $E_{0}$, lying below the $V_{m}$ nullcline. By the Poincaré-Bendixson theorem, this limit set must be an (unstable) equilibrium point, a periodic orbit or a connected set of homoclinic/heteroclinic orbits. Part 2 rules out the possibility of a periodic orbit. The only possible homoclinic orbit would need to be based at the saddle coexistence equilibrium. However, the unstable manifold of $E_{0}$ is contained within two disjoint (except for one point: $E_{0}$ itself) positively invariant sets, each of which contains a single, stable equilibrium point $\left(W_{0}(\epsilon)\right.$ and $M_{0}$ respectively). Consequently, $\alpha\left(x_{0}\right)$ cannot contain a point on the unstable manifold of $E_{0}$, since any point on this manifold has either $W_{0}(\epsilon)$ or $M_{0}$ as its $\omega$-limit set. It follows that $\alpha\left(x_{0}\right)$ does not contain a homoclinic orbit. Moreover, all equilibria excluding $E_{0}$ have all of the real parts of their eigenvalues strictly negative or strictly positive, indicating that $\alpha\left(x_{0}\right)$ does not consist of a circuit of heteroclinic orbits connecting these equilibria. The above discussion allows us to conclude that $\alpha\left(x_{0}\right)=0$. Therefore there is a heteroclinic orbit connecting $E_{0}$ with the extinction equilibrium.

\section{Proof of Theorem 7}

First, we consider the stability of equilibria of system (12) when $\epsilon=0$, under the assumptions B1-B2 of Theorem 7. Due to assumption B2, there are no coexistence equilibria. The equilibria are $\overline{0}=(0,0), W_{0}=\left(K_{w}, 0\right)$ and $M_{0}=\left(0, K_{m}\right)$, as in the proof of Theorem $6 . W_{0}$ and $\overline{0}$ are seen to be stable and hyperbolic, by considering the linearizations appearing in the proof of Theorem 6. Consequently, $W_{0}$ persists under small perturbations of $\epsilon$ and remains a sink. $M_{0}$, however, is non-hyperbolic, because of condition B2.

When $\epsilon>0$, there is a mutant-only equilibrium at

$$
M_{0}(\epsilon)=\left(0, K_{m}\right)=\left(0, \frac{K_{w}}{\alpha_{w m}} g(\epsilon)\right)
$$


This particular equilibrium is formed by the nullclines $V_{w}=0$ and $V_{w}=$ $M\left(V_{m}\right)$ (see the proof of Theorem 5). The non-hyperbolicity occurs because, at $\epsilon=0$, the nullclines $V_{w}=W\left(V_{m}\right)$ and $V_{w}=M\left(V_{m}\right)$ both intersect at $M_{0}(0)$. The nullcline $V_{w}=M\left(V_{m}\right)$ is the solution $V_{w}$ of equation (25). Restricting that equation to the nullcline $V_{w}=W\left(V_{m}\right)$ and applying assumption B2 results in

$$
V_{m}^{2}\left(\frac{r_{m} \alpha_{w m}}{g(\epsilon)}\right)+V_{m} W \cdot\left(\frac{r_{m} \alpha_{m w} \alpha_{w m}}{K_{w} g(\epsilon)}+\epsilon K_{b w m}\right)+W^{2} \epsilon K_{b w m}-\epsilon b_{w}^{0} W=0 .
$$

Let us denote the left-hand side of the above equation by $G\left(V_{m}, \epsilon\right)$. Recall now that we can express $W$ as

$$
W=-\left(\frac{\alpha_{w m} r_{m}-\epsilon K_{b w m} K_{w}}{r_{w}-\epsilon K_{b w w} K_{w}}\right) V_{m}+\frac{K_{w}\left(r_{w}-\epsilon b_{w}^{0}\right)}{\left.r_{w}-\epsilon K_{b w w} K_{w}\right)} .
$$

It follows that $G$ is $C^{1}$ and satisfies $G\left(\frac{K_{w}}{\alpha_{w m}}, 0\right)=0$. The partial derivatives are

$$
\begin{aligned}
\frac{\partial G}{\partial V_{m}}\left(\frac{K_{w}}{\alpha_{w m}}, 0\right)=r_{m} & \left(1-\frac{K_{w}}{r_{w}}\right) \\
\frac{\partial G}{\partial \epsilon}\left(\frac{K_{w}}{\alpha_{w m}}, 0\right)=- & \frac{g^{\prime}(0) K_{w}^{2} r_{m}}{\alpha_{w m}}+\frac{r_{m} \alpha_{m w} K_{w}}{r_{w}}\left(\frac{K_{w}\left(K_{b w m} r_{w}-K_{b w w} \alpha_{w m} r_{m}\right)}{\alpha_{w m} r_{w}}\right. \\
& \left.+k_{b w w} K_{w}-b_{w}^{0}\right) .
\end{aligned}
$$

By the implicit function theorem, there exists a unique $C^{1}$ function $V_{m}$ : $\epsilon \mapsto V_{m}(\epsilon)$ satisfying $V_{m}(0)=\frac{K_{w}}{\alpha_{w m}}$ and $G\left(V_{m}(\epsilon), \epsilon\right)=0$ for $|\epsilon|$ sufficiently small. It follows that $\left(W\left(V_{m}(\epsilon)\right), V_{m}(\epsilon)\right)$ is a coexistence equilibrium (i.e., is nonnegative in both components) for $\epsilon>0$ sufficiently small, provided $\frac{\partial V_{m}}{\partial \epsilon}(0)<0$. By the implicit function theorem, we have

$$
\frac{\partial V_{m}}{\partial \epsilon}(0)=-\left(\frac{\partial G}{\partial V_{m}}\left(\frac{K_{w}}{\alpha_{w m}}, 0\right)\right)^{-1} \frac{\partial G}{\partial \epsilon}\left(\frac{K_{w}}{\alpha_{w m}}, 0\right) .
$$

Equation (34), along with some straightforward algebra, shows that $\frac{\partial V_{m}}{\partial \epsilon}(0)<$ 0 is equivalent to inequality (18).

The rest of the proof proceeds similarly to that of Theorem 6 and is thus omitted. 


\section{Proof of Theorem 8}

For brevity, we will write $q^{*}=q_{0}^{*}(\epsilon)$. Let $\phi(t, q, x)$ denote the flow from time $t=0$ of the solution of the impulsive system (12), with initial condition $\phi(0, q, x)=x$ and spraying efficacy $q$. All other parameters are assumed to be fixed. Denote $N(q, x)=\phi(T, q, x)-x$. $D_{x} N(q, 0)$ is easily found to be

$$
\begin{aligned}
D_{x} N(q, 0) & =\left[\begin{array}{cc}
(1-q) e^{\left(r_{w}-\epsilon b_{w}^{0}\right) T}-1 & 0 \\
\xi & e^{r_{m} T}-1,
\end{array}\right], \\
\xi & =\frac{\epsilon b_{w}^{0}\left(e^{r_{m} T}-e^{\left(r_{w}-\epsilon b_{w}^{0}\right) T}\right)}{\left(r_{m}-r_{w}+\epsilon b_{w}^{0}\right) T} .
\end{aligned}
$$

Consequently, $\operatorname{det} D_{x} N\left(q^{*}, 0\right)=0$. Let us define the variable $\bar{q}=q-$ $q^{*}$ and define $M(\bar{q}, x)=N\left(\bar{q}+q^{*}, x\right)$, so that we have $M(0,0)=0$ and $\operatorname{det} D_{x} M(0,0)=0$. Our objective will now be to obtain a nontrivial solution of the equation $M(\bar{q}, x)=0$.

Write $M=\left[\begin{array}{ll}M_{1} & M_{2}\end{array}\right]^{T}$ in component form, and define $f: \mathbb{R} \times \mathbb{R} \times \mathbb{R} \rightarrow$ $\mathbb{R}^{2}$ by $f(\bar{q}, r, s)=M\left(\bar{q}, r e_{1}+s e_{2}\right)$, where $e_{1}$ and $e_{2}$ are the standard basis vectors in $\mathbb{R}^{2}$. Then, with $f=\left[\begin{array}{ll}f_{1} & f_{2}\end{array}\right]^{T}$, we can readily calculate the partial derivative of $f_{2}$ with respect to $s$ at 0 . We have

$$
\frac{\partial f_{2}}{\partial s}(0)=\frac{\partial M_{2}}{\partial x_{2}}(0)=e^{r_{m} T}-1 .
$$

Therefore $\frac{\partial f_{2}}{\partial s}(0) \neq 0$ and, by the implicit function theorem, there exists a unique smooth function $s:(\bar{q}, r) \mapsto s(\bar{q}, r)$ such that $f_{2}(\bar{q}, r, s(\bar{q}, r))=0$ and $s(0,0)=0$, which is defined in some neighbourhood of $(0,0)$. We also have the partial derivatives

$$
\begin{aligned}
& \frac{\partial s}{\partial r}=-\frac{\partial f_{2}}{\partial s} \frac{-1}{\partial r}=-\frac{1}{e^{r_{m} T}-1} \frac{\partial M_{2}}{\partial x_{1}}=-\frac{\xi}{e^{r_{m} T}-1} \\
& \frac{\partial s}{\partial \bar{q}}=-\frac{\partial f_{2}}{\partial s} \quad \frac{\partial f_{2}}{\partial \bar{q}}=-\frac{1}{e^{r_{m} T}-1} \frac{\partial M_{2}}{\partial \bar{q}}=0
\end{aligned}
$$

with all partial derivatives evaluated at zero, and the final equality follows from the fact that $N(q, 0)=0$, so that $M(\bar{q}, 0)=0$.

Our problem is now reduced to solving the equation $f_{1}(q, r, s(\bar{q}, r))=0$. This will be accomplished by a second-order application of Taylor's theorem. To simplify notation, write $g(\bar{q}, r)=f_{1}(q, r, s(\bar{q}, r))$, so we must solve 
$g(\bar{q}, r)=0$. The first-order partial derivatives of $g$ at zero are

$$
\begin{aligned}
\frac{\partial}{\partial r} g & =\left.\frac{\partial}{\partial r} f_{1}(0, r, s(0, r))\right|_{r=0}=\frac{\partial f_{1}}{\partial r}(0)+\frac{\partial f_{1}}{\partial s}(0) \frac{\partial s}{\partial \bar{q}}(0) \\
& =D_{x} M(0,0)_{11}+D_{x} M(0,0)_{12} \cdot\left(-\frac{\xi}{e^{r_{m} T}-1}\right)=0 \\
\frac{\partial}{\partial \bar{q}} g & =\frac{\partial N_{1}}{\partial q}+\frac{\partial N_{1}}{\partial x_{2}} \frac{\partial s}{\partial \bar{q}}=0 .
\end{aligned}
$$

We must now calculate the second-order partial derivatives of $g$. First, since $N_{1}\left(q, s e_{2}\right)=0$ for all $q, s \in \mathbb{R}$, we readily find that $\frac{\partial^{2} g}{\partial \bar{q}^{2}}$ is zero. Indeed,

$$
\frac{\partial^{2} g}{\partial \bar{q}^{2}}=\left.\frac{\partial^{2}}{\partial q^{2}} N_{1}\left(q, s\left(q-q^{*}, 0\right) e_{2}\right)\right|_{q=q^{*}}=0 .
$$

The other second-order partial derivatives require a bit more effort. We find

$$
\begin{aligned}
\frac{\partial^{2} g}{\partial r^{2}} & =\partial_{x_{1} x_{1}} N_{1}+\partial_{x_{2} x_{1}} N_{1} \cdot \partial_{r} s+\partial_{x_{2} x_{1}} N_{1} \cdot \partial_{r} s+\partial_{x_{2} x_{2}} N_{1} \cdot \partial_{r} s+\partial_{x_{2}} N_{1} \cdot \partial_{r r} s \\
& =\partial_{x_{1} x_{1}} N_{1}+\partial_{r} s \cdot\left(2 \partial_{x_{2} x_{1}} N_{1}+\partial_{x_{2} x_{2}} N_{1}\right)
\end{aligned}
$$

where $\partial_{x_{2}} N_{1}=0$ is known by previous calculation. Notice, however, that since $\partial_{r} s=-\xi /\left(e^{r_{m} T}-1\right)$ and $\xi=\epsilon C(\epsilon)$ is continuous at $\epsilon=0$, it follows that $\partial_{r} s=0$ when $\epsilon=0$. Therefore the sign of $\frac{\partial^{2} g}{\partial r^{2}}$ near $\epsilon=0$ is determined primarily by the sign of $\partial_{x_{1} x_{1}} N_{1}$. We therefore compute only this partial derivative.

The partial derivatives of $N$ seen above can be determined by solving certain differential equations. The following are true about the function $z(t, x)=\phi_{1}\left(t, q^{*}, x\right)$ :

$$
\begin{aligned}
\frac{d}{d t} \partial_{x_{1} x_{1}} z & =\left(r_{w}-\epsilon b_{w}^{0}\right) \partial_{x_{1} x_{1}} z+\left[\left(1-q^{*}\right) e^{\left(r_{w}-\epsilon b_{w}^{0}\right) t}\right]^{2}\left(2 \epsilon K_{b w w}-\frac{2 r_{w}}{K_{w}}\right), \\
\partial_{x_{1} x_{1}} z(0) & =0 .
\end{aligned}
$$

The above can be computed analytically, and we find

$$
\partial_{x_{1} x_{1}} z(T)=\frac{2 e^{\left(r_{w}-\epsilon b_{w}^{0}\right) T}\left(e^{\left(r_{w}-\epsilon b_{w}^{0}\right) T}-1\right)}{r_{w}-\epsilon b_{w}^{0}}\left(\epsilon K_{b w w}-\frac{r_{w}}{K_{w}}\right)<0,
$$


where the inequality holds provided $|\epsilon|$ is sufficiently small. By (35), it follows that $\frac{\partial^{2} g}{\partial r^{2}}(0)<0$ when $|\epsilon|$ is small enough.

Next we calculate the mixed partial derivative in the variables $\bar{q}$ and $r$. We find

$$
\begin{aligned}
\frac{\partial^{2} g}{\partial \bar{q} r} & =\partial_{q x_{1}} N_{1}+\partial_{x_{2} x_{1}} N_{1} \cdot \partial_{\bar{q}} s+\partial_{q x_{2}} N_{1} \cdot \partial_{r} s+\partial_{x_{2} x_{2}} N_{1} \cdot\left(\partial_{r} s\right)^{2}+\partial_{x_{2}} N_{1} \partial_{\bar{q} r} s \\
& =\partial_{q x_{1}} N_{1}
\end{aligned}
$$

as almost all partial derivatives vanish.

As before, $\partial_{q x_{2}} N_{1}$ can be found by solving a particular differential equation. However, we do not have to, since it is already known that $\partial_{x_{1}} N_{1}(q, 0)=$ $(1-q) e^{\left(r_{w}-\epsilon b_{w}^{0}\right) T}-1$. Therefore we have

$$
\partial_{q x_{1}} N_{1}=\left.\frac{\partial}{\partial q} \partial_{x_{1}} N_{1}(q, 0)\right|_{q=q^{*}}=-e^{\left(r_{w}-\epsilon b_{w}^{0}\right) T}<0 .
$$

Hence, by (36), we have $\frac{\partial^{2} g}{\partial \bar{q} r}<0$.

From the above calculations and Taylor's theorem, we have

$$
g(\bar{q}, r)=A r^{2}+B \bar{q} r+o\left(\bar{q}^{2}+r^{2}\right)
$$

as $(\bar{q}, r) \rightarrow 0$, for real numbers $A=\frac{1}{2} \partial_{r^{2}} g(0)$ and $B=\partial_{\bar{q} r} g(0)$ satisfying $A, B<0$. Near $(\bar{q}, r)=0$, there is a nontrivial branch of solutions, written approximately as

$$
r(q) \approx-\frac{B}{A} \bar{q}(q)=-\frac{B}{A}\left(q-q^{*}\right)
$$

Therefore the initial condition of our nontrivial periodic solution can be written, locally, as the approximation

$$
p(T, q) \approx\left(-\frac{B}{A}\left(q-q^{*}\right), s\left(q-q^{*},-\frac{B}{A}\left(q-q^{*}\right)\right)\right) .
$$

To determine the location of this point as $q$ varies near $q^{*}$, we calculate partial derivatives, considering, for this purpose, the above approximation to be exact (as it is essentially a linear approximation). We have

$$
\frac{\partial p}{\partial q}\left(T, q^{*}\right)=\left(-\frac{B}{A}, \partial_{\bar{q}} s(0)-\frac{B}{A} \partial_{r} s(0)\right)=\left(-\frac{B}{A}, \frac{B \xi}{A\left(e^{r_{m} T}-1\right)}\right),
$$

whose components have sign $(-,+)$. It follows that $p(T, q)$ is nonpositive for all $q$ sufficiently close to $q^{*}$, while $p\left(T, q^{*}\right)=0$. This proves the theorem. 


\section{Proof of Theorem 9}

The proof will follow much the same format as that of Theorem 8. As before, let $\phi(t, q, x)$ denote the flow from time $t=0$ of the solution of the impulsive system (12), with initial condition $\phi(0, q, x)=x$ and spraying efficacy $q$. All other parameters are assumed to be fixed. Denote $N(q, x)=\phi(T, q, x)-x$, $M_{0}=\left(0, K_{m}\right)$ and $q^{*}=q_{M}^{*}(\epsilon) . D_{x} N\left(q, M_{0}\right)$ is easily found to be

$$
\begin{gathered}
D_{x} N\left(q, M_{0}\right)=\left[\begin{array}{cc}
(1-q) \exp \left(\left(r_{w}\left(1-\frac{\alpha_{w m} K_{m}}{K_{w}}\right)-\epsilon\left(b_{w}^{0}-K_{b w m} K_{m}\right)\right) T\right)-1 & 0 \\
\chi & e^{-r_{m} T}-1,
\end{array}\right], \\
\chi=\chi(\epsilon)=\frac{\left(-r_{m} \alpha_{m w}+\epsilon b_{w}^{0}\right)\left[e^{-r_{m} T}-\exp \left(\left(r_{w}\left(1-\frac{\alpha_{w m} K_{m}}{K_{w}}\right)-\epsilon\left(b_{w}^{0}-K_{b w m} K_{m}\right)\right) T\right)\right]}{-\left(r_{m}+r_{w}\left(1-\frac{\alpha_{w m} K_{m}}{K_{w}}\right)-\epsilon\left(b_{w}^{0}-K_{b w m} K_{m}\right)\right) T} .
\end{gathered}
$$

Consequently, $\operatorname{det} D_{x} N\left(q^{*}, M_{0}\right)=0$.

As before, define $\bar{q}=q-q^{*}$ and define the function $f=\left(f_{1}, f_{2}\right): \mathbb{R}^{3} \rightarrow \mathbb{R}^{2}$ by

$$
f(\bar{q}, r, s)=N\left(\bar{q}+q^{*}, r e_{1}+\left(s+M_{0}\right) e_{2}\right) .
$$

The first-order partial derivatives of $f$ are very similar to those appearing in the proof of Theorem 8; we state the results without proof. All partial derivatives are calculated at zero.

$$
\frac{\partial f_{2}}{\partial s}=e^{-r_{m} T}-1, \quad \frac{\partial f_{2}}{\partial \bar{q}}=0, \quad \frac{\partial f_{2}}{\partial r}=\chi .
$$

Therefore, as before, we apply the implicit function theorem to write $s=$ $s(\bar{q}, r)$. If we define $g(\bar{q}, r)=f_{1}(\bar{q}, r, s(\bar{q}, r))$, then the partial derivatives at zero satisfy

$$
\frac{\partial g}{\partial \bar{q}}=\frac{\partial g}{\partial r}=0
$$

Also, the implicit function $s$ satisfies

$$
\frac{\partial s}{\partial \bar{q}}(0)=0, \quad \frac{\partial s}{\partial r}(0)=\frac{\chi}{1-e^{-r_{m} T}}
$$

As for the second-order partial derivatives at zero, we find $\frac{\partial^{2} g}{\partial \bar{q}^{2}}(0)=0$, since $N_{1}\left(q, M_{0}\right)=0$ for all $q$. The symbolic calculation of the other partial 
derivatives are the same as in the previous theorem; in particular, all partial derivatives appearing in $\frac{\partial^{2} g}{\partial \bar{q} r}$ vanish except for one:

$\frac{\partial^{2} g}{\partial \bar{q} r}=\partial_{q x_{1}} N\left(q^{*}, M_{0}\right)=\left.\frac{\partial}{\partial q} D_{x} N\left(q, M_{0}\right)_{11}\right|_{q=q^{*}}=-e^{\left(r_{w}\left(1-\frac{\alpha_{w m} K_{m}}{K_{w}}\right)-\epsilon\left(b_{w}^{0}-K_{b w m} K_{m}\right)\right) T}<0$.

For the double partial derivative in the variable $r$, not as many terms are able to be ignored as in the previous proof, since $\chi$ does not become negligible as $\epsilon$ becomes small. Comparing to the first line of (35), many terms do indeed vanish (any term involving only $N_{1}$ without partial derivatives in $x_{1}$ will vanish). We find

$$
\frac{\partial^{2} g}{\partial r^{2}}=\partial_{x_{1} x_{1}} N_{1}\left(q^{*}, M_{0}\right)+\left(2 \partial_{r} s\right) \cdot \partial_{x_{1} x_{2}} N_{1}\left(q^{*}, M_{0}\right)
$$

The mixed partial derivative can be found by solving the differential equation

$$
\begin{aligned}
\frac{d}{d t} \partial_{x_{2} x_{1}} z & =A \partial_{x_{1} x_{2}} z+\left(1-q^{*}\right) \cdot\left(\epsilon K_{b w m}-\frac{r_{w} \alpha_{w m}}{K_{w}}\right) e^{\left(A-r_{m}\right) t}, \\
A & =A(\epsilon)=r_{w}\left(1-\frac{\alpha_{w m} K_{m}}{K_{w}}\right)-\epsilon\left(b_{w}^{0}-K_{b w m} K_{m}\right), \\
\partial_{x_{2} x_{1}} z(0) & =0 .
\end{aligned}
$$

Specifically, we have $\partial_{x_{2} x_{1}} z(T)=\partial_{x_{2} x_{1}} \phi\left(T, q^{*}, M_{0}\right)=\partial_{x_{2} x_{1}} N_{1}\left(q^{*}, M_{0}\right)$. Computing, we find, since $e^{A(\epsilon) T}=\frac{1}{1-q^{*}}$, that

$$
\partial_{x_{2} x_{1}} N_{1}\left(q^{*}, M_{0}\right)=\left(1-e^{-r_{m} T}\right) \frac{\epsilon K_{b w m}-\frac{r_{w} \alpha_{w m}}{K_{w}}}{r_{m}} .
$$

The double partial derivative in $x_{1}$ can calculated similarly: when $\epsilon=0$, $\partial_{x_{1} x_{1}} N_{1}\left(q^{*}, M_{0}\right)=\partial_{x_{1} x_{1}} w(T)$, where

$$
\begin{aligned}
\frac{d}{d t} \partial_{x_{1} x_{1}} w & =A(0) \partial_{x_{1} x_{1}} w-\frac{2 r_{w}}{K_{w}} e^{2 A(0) t}\left[1+\frac{\alpha_{w m} \alpha_{m w} r_{m}}{r_{m}+A(0)}\left(e^{A(0) t}-e^{-r_{m} t}\right)\right] \\
\partial_{x_{1} x_{1}} w(0) & =0 .
\end{aligned}
$$

Therefore,

$\partial_{x_{1} x_{1}} N_{1}\left(q^{*}, M_{0}\right)=-\frac{2 r_{w}}{K_{w}} e^{A(0) T} \int_{0}^{T} e^{A(0) s}\left[1+\frac{\alpha_{w m} \alpha_{m w} r_{m}}{r_{m}+A(0)}\left(e^{A(0) s}-e^{-r_{m} s}\right)\right] d s$ 
In summary, when $\epsilon=0$, we have the following conclusions:

$$
\begin{aligned}
\frac{\partial^{2} g}{\partial \bar{q} r} & =-\frac{1}{1-q_{M}^{*}(0)}<0 \\
\frac{\partial^{2} g}{\partial \bar{q}^{2}} & =0 \\
\frac{\partial^{2} g}{\partial r^{2}} & =-\frac{2 r_{w}}{K_{w}} e^{A(0) T} \int_{0}^{T} e^{A(0) s}\left[1+\frac{\alpha_{w m} \alpha_{m w} r_{m}}{r_{m}+A(0)}\left(e^{A(0) s}-e^{-r_{m} s}\right)\right] d s-2 \chi(0) \frac{r_{w} \alpha_{w m}}{r_{m} K_{w}} \\
& =-\frac{2 r_{w}}{K_{w}}(Y+\chi(0) Z)
\end{aligned}
$$

Under the assumptions of the theorem, we have $Y+\chi(0) Z>0$. The rest of the proof is now essentially identical to the proof of Theorem 8 and is hence omitted.

\section{Proof of Theorem 10}

Let $\phi(t, x, q, \epsilon)$ denote the flow from time $t=0$ of the solution of the impulsive system (12), with initial condition $\phi(0, x, q, \epsilon)=x$, spraying efficacy $q$ and mutation rate $\epsilon$. All other parameters are assumed to be fixed. Denote $N(x, q, \epsilon)=\phi(T, x, q, \epsilon)-x$.

A straightforward calculation shows that, for an initial condition of the form $x_{w}=\left(V_{w}, V_{m}\right)=\left(x_{w}, 0\right)$, the differential $D_{x} N\left(x_{w}, q, 0\right)$ can be written

$$
\begin{aligned}
D_{x} N\left(x_{w}, q, 0\right) & =\left[\begin{array}{cc}
(1-q) e^{r_{w} T}\left[Z\left(x_{w}\right)\right]^{-2}-1 & \frac{-\alpha_{w m} e^{r_{w} T}\left[Z\left(x_{w}\right)\right]^{-2}\left(1-\left[Z\left(x_{w}\right)\right]^{-2-Y}\right)}{2+Y} \\
0 & e^{r_{m} T}\left[Z\left(x_{w}\right)\right]^{-Y}-1
\end{array}\right] \\
Z\left(x_{w}\right) & =\frac{K_{w}+x_{w}\left(e^{r_{w} T}-1\right)}{K_{w}} \\
Y & =\frac{r_{m} \alpha_{m w} K_{w}}{r_{m} K_{m}} .
\end{aligned}
$$

It is easy to verify that $D_{x}\left(w_{0}\left(q_{2}^{*}\right), q_{2}^{*}, 0\right)$ is non-invertible and has the form $D_{x} N\left(w_{0}\left(q_{2}^{*}\right), q_{2}^{*}, 0\right)=\left[\begin{array}{cc}A & B \\ 0 & 0\end{array}\right]$. Note that $A \neq 0$.

Define the map $L: \mathbb{R}^{3+1} \rightarrow \mathbb{R}^{3}$ by $L(x, q ; \epsilon)=\left[N(x, q, \epsilon) \operatorname{det} D_{x} N(x, q, \epsilon)\right]^{T}$. 
From the above, we know that $D_{x, \epsilon} L\left(w_{0}\left(q_{2}^{*}\right), q_{2}^{*}, 0\right)$ has the structure

$$
\begin{aligned}
D_{x, \epsilon} L\left(w_{0}\left(q_{2}^{*}\right), q_{2}^{*}, 0\right) & =\left[\begin{array}{ccc}
A & B & \cdots \\
0 & 0 & E \\
C & D & \cdots
\end{array}\right], \\
{\left[\begin{array}{cc}
A & B
\end{array}\right] } & =D_{x} N\left(w_{0}\left(q_{2}^{*}\right), q_{2}^{*}, 0\right) e_{1}, \\
{\left[\begin{array}{cc}
C & D
\end{array}\right] } & =\left[\nabla_{x} \operatorname{det} D_{x}\right] N\left(w_{0}\left(q_{2}^{*}\right), q_{2}^{*}, 0\right), \\
E & =\frac{\partial N_{2}}{\partial \epsilon}\left(w_{0}\left(q_{2}^{*}\right), q_{2}^{*}, 0\right) .
\end{aligned}
$$

It follows that $D_{x, \epsilon} L\left(w_{0}\left(q_{2}^{*}\right), q_{2}^{*}, 0\right)$ is invertible, provided $E \neq 0$ and $A D-$ $B C \neq 0$. If this is the case, the implicit function theorem will guarantee the existence of a function satisfying all the conditions of Theorem 10 except for the condition on the partial derivative of $\epsilon(q)$. We will prove this part later.

Calculation of $E$. We have

$$
\frac{d}{d t} \frac{\partial \phi_{2}}{\partial \epsilon}\left(t, w_{0}\left(q_{2}^{*}\right), q_{2}^{*}, \epsilon\right)=\frac{\partial}{\partial \epsilon}\left[\epsilon \tilde{w}\left(t, q_{2} *\right)\left(b_{w}^{0}-K_{b w w} \tilde{w}\left(t, q_{2}^{*}\right)\right],\right.
$$

so that

$$
\frac{d}{d t} \frac{\partial \phi_{2}}{\partial \epsilon}\left(t, w_{0}\left(q_{2}^{*}\right), q_{2}^{*}, 0\right)=\tilde{w}\left(t, q_{2}^{*}\right)\left(b_{w}^{0}-K_{b w w} \tilde{w}\left(t, q_{2}^{*}\right)\right) .
$$

From this, we conclude that

$$
E=\frac{\partial N_{2}}{\partial \epsilon}\left(w_{0}\left(q_{2}^{*}\right), q_{2}^{*}, 0\right)=\int_{0}^{T} \tilde{w}\left(t, q_{2}^{*}\right)\left(b_{w}^{0}-K_{b w w} \tilde{w}\left(t, q_{2}^{*}\right)\right) d t .
$$

Since we are working in one of the biologically relevant domains described in Theorem 4, we know that $b_{w}^{0}>K_{b w w} \tilde{w}\left(t, q_{2}^{*}\right)$ for all $t \in[0, T]$ because $\tilde{w}\left(t, q_{2}^{*}\right)>0$. Consequently, the integrand above is strictly positive, and we conclude that $E>0$.

Simplification of $A D-B C$. By Jacobi's formula, [ $\left.\begin{array}{ll}C & D\end{array}\right]$ can be written

$\left[\begin{array}{ll}C & D\end{array}\right]=\nabla_{x} \operatorname{det} D_{x}\left(w_{0}\left(q_{2}^{*}\right), q_{2}^{*}, 0\right)=\left[\begin{array}{c}\operatorname{tr}\left(\operatorname{adj}\left(L_{1}\right) \frac{d}{d_{V_{w}}} \circ D_{x} N\left(w_{0}\left(q_{2}^{*}\right), q_{2}^{*}, 0\right)\right) \\ \operatorname{tr}\left(\operatorname{adj}\left(L_{1}\right) \frac{d}{d_{V_{m}}} \circ D_{x} N\left(w_{0}\left(q_{2}^{*}\right), q_{2}^{*}, 0\right)\right)\end{array}\right]^{T}$, 
where $L_{1}=D_{x} N\left(w_{0}\left(q_{2}^{*}\right), q_{2}^{*}, 0\right)=\left[\begin{array}{cc}A & B \\ 0 & 0\end{array}\right]$. However, it can be shown that $M \equiv \frac{d}{d V_{w}} \circ D_{x} N\left(w_{0}\left(q_{2}^{*}\right), q_{2}^{*}, 0\right)$ is upper triangular. Consequently,

$$
C=\operatorname{tr}\left(\operatorname{adj}\left(L_{1}\right) M\right)=A \cdot M_{22} \text {. }
$$

Taking into account that $A \neq 0$, we have $A D-B C \neq 0$ if and only if $D-M_{22} B \neq 0$.

Calculation of $M_{22}$. We have $M_{22}=\left.\frac{d}{d x_{w}} D_{x}\left(x_{w}, q_{2}^{*}, 0\right)_{22}\right|_{x_{w}=w_{0}\left(q_{2}^{*}\right)}$. The matrix $D_{x}\left(x_{w}, q, 0\right)$ appears in equation (37). We have

$$
M_{22}=-Y e^{r_{m} T} Z^{\prime}\left(w_{0}\left(q_{2}^{*}\right)\right)\left[Z\left(w_{0}\left(q_{2}^{*}\right)\right)\right]^{-Y-1}=\frac{1}{K_{w}} e^{-r_{w} T}\left(e^{r_{w} T}-1\right)=\frac{q_{1}^{*}}{K_{w}},
$$

where the final two equalities result from straightforward algebra.

Calculation of $D$. It can be shown that $Z(t) \equiv \frac{d}{d V_{m}} D_{x} \phi\left(t, w_{0}\left(q_{2}^{*}\right), q_{2}^{*}, 0\right)$ satisfies the set of matrix initial-value problems

$$
\begin{aligned}
& Z^{\prime}=Q(t) Z+R(t) S(t), \\
& S^{\prime}=Q(t) S \text {, } \\
& t \neq k T \\
& \Delta Z=\left[\begin{array}{cc}
0 & 0 \\
0 & -q_{2}^{*}
\end{array}\right] Z, \\
& t \neq k T \\
& \Delta S=\left[\begin{array}{cc}
0 & 0 \\
0 & -q_{2}^{*}
\end{array}\right] S, \\
& t=k T \\
& Z(0)=0 \text {, } \\
& S(0)=I \text {, } \\
& Q(t)=\left[\begin{array}{cc}
r_{w}\left(1-\frac{2 \tilde{w}\left(t, q_{2}^{*}\right)}{K_{w}}\right) & -\frac{r_{w} \alpha_{w m}}{K_{w}} \tilde{w}\left(t, q_{2}^{*}\right) \\
0 & r_{m}\left(1-\frac{\alpha_{m w} \tilde{w}\left(t, q_{2}^{*}\right)}{K_{m}}\right)
\end{array}\right], \\
& R(t)=-e^{r_{m} t}\left[\begin{array}{cc}
\frac{r_{w} \alpha w m}{K_{w}} & 0 \\
\frac{r_{m} \alpha_{m w}}{K_{m}} & \frac{2 r_{m}}{K_{m}}
\end{array}\right] \\
& t=k T
\end{aligned}
$$

As such, if we denote by $\mathbf{S}(t)$ the fundamental matrix solution of the system $S^{\prime}=Q(t) S$ satisfying $\mathbf{S}(0)=I$, we can write, by the variation of constants formula,

$$
H \equiv \frac{d}{d V_{m}} D_{x} \phi\left(T, w_{0}\left(q_{2}^{*}\right), q_{2}^{*}, 0\right)=\mathbf{S}(T) \int_{0}^{T} \mathbf{S}^{-1}(t) R(t)\left[\begin{array}{cc}
1-q_{2}^{*} & 0 \\
0 & 1
\end{array}\right] \mathbf{S}(t) d t
$$


we have

Now, since $\frac{d}{d V_{m}} N(x, q, \epsilon)=\frac{d}{d V_{m}}\left(D_{x} \phi(T, x, q, \epsilon)-I\right)=\frac{d}{d V_{m}} D_{x} \phi(T, x, q, \epsilon)$,

$$
D=\operatorname{tr}\left(\left[\begin{array}{cc}
0 & -B \\
0 & A
\end{array}\right] \cdot H\right)=\operatorname{tr}\left[\begin{array}{cc}
-B H_{21} & \cdots \\
\cdots & A H_{22}
\end{array}\right]=-B H_{21}+A H_{22} .
$$

An elementary calculation shows that $H_{21}=-\left(1-q_{2}^{*}\right) \frac{\alpha_{m w}}{K_{m}}\left(e^{r_{m} T}-1\right)$ and $H_{22}=-\frac{2}{K_{m}}\left(e^{r_{m} T}-1\right)$.

Conclusion. The value of $B$ cannot be written in terms of elementary functions unless $r_{w}=r_{m}$. The condition that $-B H_{21}+A H_{22}-M_{22} B=$ $A H_{22}-B\left(H_{21}+M_{22}\right) \neq 0$ is equivalent to condition (20) of the theorem.

\section{Proof of Lemma 5}

The mosquito compartment has a unique nontrivial periodic solution,

$$
\begin{aligned}
\tilde{\Psi}(t ; T) & =\frac{K_{w} e^{r_{w} t} w_{0}(T)}{K_{w}+\left(e^{r_{w} t}-1\right) w_{0}(T)}, \\
w_{0}(T) & =\frac{K_{w}\left(e^{r_{w} T}(1-q)-1\right)}{e^{r_{w} T}-1} .
\end{aligned}
$$

It follows that if $T>T_{*}$, then $\tilde{\Psi}>0$. Therefore, to search for nontrivial periodic solutions of $(21)$, we may take $\Psi=\tilde{\Psi}$. We must prove the existence of positive periodic solutions of

$$
\begin{aligned}
\dot{S} & =\pi-\beta_{H} S \tilde{\Psi}+h I+\delta R-\mu_{H} S, \\
\dot{I} & =\beta_{H} S \tilde{\Psi}-h I-\alpha I-\gamma I-\mu_{h} I, \\
\dot{R} & =\alpha I-\delta R-\mu_{H} R .
\end{aligned}
$$

However, this ordinary differential equation is linear, $R_{+}^{3}$ is positively invariant, and the sum $H=S+I+R$ satisfies

$$
\dot{H} \leq \pi-\mu_{H} H
$$

for positive initial conditions, implying that this linear system has a bounded solution. By the Massera theorem [11], it has a periodic solution.

Conversely, if $T \leq T_{*}$, the only nonnegative recurrent state of the mosquito dynamics is the extinction equilibrium, which is globally asymptotically stable for nonnegative initial population. A comparison principle argument then shows that the disease-free equilibrium is globally attracting. 


\section{Proof of Lemma 6}

As before, we may take $\Psi=\tilde{\Psi}$. Under the assumptions of the lemma, $H=S+I+R$ satisfies the differential equation $H^{\prime}=\pi-\mu_{H} H$. Therefore, in searching for periodic solutions, we may assume $H=\frac{\pi}{\mu_{H}}$ is constant. Consequently,

$$
\beta(P)=\frac{\beta_{H} \Psi}{S+I+R}=\frac{\beta_{H} \mu_{H}}{\pi} \Psi .
$$

The result follows by Lemma 5 .

\section{Proof of Theorem 11}

We prove the theorem only for standard incidence, $\beta(P)=\beta_{H} \frac{\Psi}{S+I+R}$, since the proof for mass action is very similar. This will be proven in several parts.

Part 1: We start with the case where $\gamma=h=\alpha=0$. As in the previous proof, we may assume that $H=S+I+R$ is constant. In this case, (38) reduces to

$$
\begin{aligned}
\dot{S} & =\pi-\frac{\beta_{H}}{H} S \tilde{\Psi}+\delta R-\mu_{H} S, \\
\dot{I} & =\frac{\beta_{H}}{H} S \tilde{\Psi}-\mu_{H} I, \\
\dot{R} & =-\delta R-\mu_{H} R .
\end{aligned}
$$

The homogeneous equation associated to the above linear system has no periodic solutions and, consequently, the inhomogeneous equation has a unique periodic solution. It follows that $(21)$ has a unique periodic solution.

Part 2: Suppose $\gamma=0$. We wish to establish conditions under which any given periodic solution

$$
(\tilde{S}(t), \tilde{I}(t), \tilde{R}(t), \tilde{\Psi}(t))
$$

is nonnegative for all time. Since the positive orthant is positively invariant, it suffices to show that the initial conditions on this periodic orbit are positive. We clearly have $\tilde{\Psi}(t)>0$, and, by using the identity $\tilde{S}+\tilde{I}+\tilde{R}=\frac{\pi}{\mu_{H}}$, we find

$\tilde{S}(t)=\exp \left(-\int_{0}^{t} a(s) d s\right)\left[\tilde{S}(0)+\int_{0}^{t}\left(\pi\left(1+\frac{h}{\mu_{H}}\right)+\tilde{R}(s)(\delta-h)\right) \exp \left(\int_{0}^{s} a(w) d w\right) d s\right]$

where

$$
a(s)=\frac{\beta_{H}}{H} \tilde{\Psi}(s)+h+\mu_{H}
$$


The initial condition $\tilde{S}(0)$ can be shown to be positive provided $\tilde{R}(t)(\delta-h) \geq$ 0 . By taking $\delta=h$, this condition is satisfied. Therefore if $\delta=h$, then $\tilde{S}(t)>0$.

Conversely, if $\tilde{S}(t)>0$, then it is simple to verify by the variation of constants formula that $\tilde{I}(t)>0$ and, following this, that $\tilde{R}(t)>0$, where this result does not depend on the parameters $\delta, \gamma$ and $h$. Therefore, in conclusion, the conditions $\gamma=0$ and $\delta=h$ are sufficient, but not necessary, to guarantee positivity of any periodic solution. Moreover, if $\tilde{S}(0)>0$, then the periodic solution is positive, independent of those three parameters.

Part 3: Define the multiparameter $\lambda \equiv(\gamma, \delta, h, \alpha)$ and let $\varphi(t)$ denote the periodic solution when $\lambda=0$. By Parts 1 and 2, we have $\tilde{R}=0$ and $\tilde{S}(t)>0$ on this solution, so the solution is nonnegative. It is clear that the vector field is sufficiently regular to perform a linearization at the periodic orbit. When $\lambda=0$, the linearized equation is

$$
\begin{aligned}
\dot{u} & =\left[\begin{array}{cccc}
-\frac{\beta_{H}}{H} \tilde{\Psi}(t)-\mu_{H} & 0 & 0 & -\frac{\beta_{H}}{H} \varphi_{S}(t) \\
\frac{\beta_{H}}{H} \tilde{\Psi}(t) & -\mu_{H}-\alpha & 0 & \frac{\beta_{H}}{H} \varphi_{S}(t) \\
0 & \alpha & -\mu_{H} & 0 \\
0 & 0 & 0 & -\mu
\end{array}\right] u, \quad t \neq k T, \\
\Delta u & =\left[\begin{array}{cccc}
0 & 0 & 0 & 0 \\
0 & 0 & 0 & 0 \\
0 & 0 & 0 & 0 \\
0 & 0 & 0 & -r
\end{array}\right] u^{-},
\end{aligned}
$$

This linear system has no nontrivial periodic solutions, and the Floquet multipliers are found to be exponentials of the diagonal entries multiplied by $T$ (with one multiplier scaled by a factor of $1-r$ ), and, since these diagonal entries are negative, all multipliers are within the unit circle. Consequently [4], the periodic orbit is exponentially stable in the nonlinear system. Moreover, for $|\lambda|<\epsilon$ sufficiently small, there is a unique exponentially stable periodic solution $\varphi_{\lambda}$ that converges to $\varphi$ as $\lambda \rightarrow 0$ in the $B$ topology. In other words, the unique periodic solution persists for $\lambda \approx 0$ and is close to $\varphi$.

Let $\tilde{S}_{\lambda}(t)$ denote the $S$ component of the periodic solution at parameter $\lambda$. Since $\tilde{S}_{0}(t)>0$, the above convergence result implies that $\tilde{S}_{\lambda}(t)>0$ for $\lambda \approx 0$. By Part $2, \tilde{S}_{\lambda}(t)>0$ is a sufficient condition for nonnegativity of the periodic solution. 


\section{Proof of Theorem 12}

We will assume that we are in Case 2, since Case 1 is similar. Define the $\operatorname{map} X:\left(T_{*}, \infty\right) \rightarrow \mathbb{R}_{+}$,

$$
X(T)=\int_{0}^{T} \tilde{S}_{T}(t) \tilde{\Psi}_{T}(t) d y,
$$

where $\tilde{S}_{T}(t)$ and $\tilde{\Psi}_{T}(t)$ are the $S$ and $\Psi$ components of the unique $T$-periodic solution of the fixed-impulse equation. Note that $\dot{S} \geq \pi-\beta_{H} C S-\mu_{H} S$ for all $T>T_{*}$, where $C$ is a constant that depends on the choice of $\beta$ (mass action or standard incidence). In particular, there exists a neighbourhood of $S=0$ where $\dot{S}>0$. Hence we must have $S_{\min } \equiv \inf _{T>0}\left\|\tilde{S}_{T}\right\|_{\infty}>0$. Consequently,

$$
0<S_{\min } \int_{0}^{T} \tilde{\Psi}_{T}(t) d t \leq X(T) .
$$

The lower bound can easily be seen to approach infinity with $T \rightarrow \infty^{1}$ and so $X$ is unbounded. Since the branch of periodic solutions depends continuously on $T$ and is bounded for all $T$ and $\tilde{\Psi}_{T_{*}}=0$, it follows (by Lebesgue's dominated convergence theorem) that $R$ is continuous and $R\left(T_{*}^{+}\right)=0$. Due to the unboundedness, $R$ is surjective. From the assumptions of the theorem, the map $\eta \beta(\cdot)$ is linear, so the map $T \mapsto \int_{0}^{T} \eta \beta\left(\tilde{P}_{T}\right) \tilde{S}_{T} d t=\eta X(T)$ is also surjective. Therefore, for all $\bar{\Theta}>0$, there exists $\bar{T}$ such that

$$
\int_{0}^{\bar{T}} \eta \beta\left(\tilde{P}_{\bar{T}}\right) \tilde{S}_{\bar{T}} d t=\bar{\Theta} .
$$

Consequently, for all $\bar{\Theta}>0$, the system with autonomous spraying (22) has a periodic solution.

Denote $H=\pi / \mu_{H}$. To show uniqueness for small values of $\bar{\Theta}$, we require several auxillary results. We claim $X$ is differentiable. To show this, consider the differential equation

$$
\begin{aligned}
\dot{S} & =\pi-\frac{\beta_{H}}{H} S \bar{\Psi}_{T}(t)+h I+\delta R-\mu_{H} S \\
\dot{I} & =\frac{\beta_{H}}{H} \bar{\Psi}_{T}(t) S(t)-\alpha I-h I-\mu_{H} I \\
\dot{R} & =\alpha I-\delta R-\mu_{H} R,
\end{aligned}
$$

\footnotetext{
${ }^{1}$ Indeed, $\tilde{\Psi}_{T}(t) \rightarrow \tilde{\Psi}_{\infty}(t) \geq K_{w}(1-q)$.
} 
where $\bar{\Psi}: \mathbb{R} \times \mathbb{R} \rightarrow \mathbb{R}$ is a $C^{1}$ extension of $\Psi$ 's restriction to the interval $(0, T]$. That is, $\bar{\Psi}(t ; T)=\tilde{\Psi}_{T}(t)$ for $t \in(0, T]$. The derivative of $\bar{\Psi}(t ; T)$ with respect to $t$ when $t \leq 0$ is defined to be constant and equal to $\lim _{t \rightarrow 0+} \Psi_{T}(t)$, with an analogous definition at times $t>T$.

For every $T>T_{*}$, the human components of the periodic solution of the impulsive differential equation $(21)$, denoted $\tilde{S}_{T}(t), \tilde{I}_{T}(t)$ and $\tilde{R}_{T}(t)$, coincide with the forward solution of (40) from $S(0)=\tilde{S}_{T}(0), I(0)=\tilde{S}_{T}(0)$, $R(0)=\tilde{R}_{T}(0)$ for $t<T$. By standard results of smooth dependence on initial conditions and parameters, the solution map $x\left(t ; x_{0}, T\right)$ associated to $(40)$ is a continuously differentiable function of $T$. By the hyperbolicity hypothesis of the theorem, $\tilde{S}_{T}(0), \tilde{I}_{T}(0)$ and $\tilde{R}_{T}(0)$ are differentiable with respect to $T$ for $T-T_{*}>0$ small. The differential equation is $C^{1}$, so we have smooth dependence on initial conditions and parameters. It then follows that the composition

$$
x\left(t ; \tilde{x}_{0}(T), T\right),
$$

where $\tilde{x}_{0}(T) \equiv\left(\tilde{S}_{T}(0), \tilde{I}_{T}(0), \tilde{R}_{T}(0)\right)$, is $C^{1}$ in $T$ small for each fixed $t$. By the Leibniz integral formula [8], the map $\bar{X}: \mathbb{R}_{+} \backslash 0 \rightarrow \mathbb{R}_{+}$defined by

$$
\bar{X}(T)=\int_{0}^{T} x^{1}\left(t ; \tilde{x}_{0}(T), T\right) \bar{\Psi}_{T}(t) d t
$$

is continuously differentiable for small $T-T_{*}>0$, where the superscript $x^{1}$ denotes the first component. Since $x^{1}\left(t ; \tilde{x}_{0}(T), T\right)=\tilde{S}_{T}(t)$ and $\bar{\Psi}_{T}(t)=$ $\tilde{\Psi}_{T}(t)$ for $t<T$, we have $\bar{X}=X$. We therefore conclude that $X$ is $C^{1}$ for $T-T_{*}>0$ small.

Next we claim that $\lim _{T \rightarrow T_{*}^{+}} X^{\prime}(T) \equiv X^{\prime}\left(T_{*}^{+}\right)$exists and is zero. Again, first considering the map $\bar{X}$, by the Leibniz integral formula [8], the limit $\overline{X^{\prime}}\left(T_{*}^{+}\right)$is given by

$$
\begin{aligned}
\lim _{T \rightarrow T_{*}^{+}} \int_{0}^{T}\left[\frac{d}{d T} \bar{\Psi}(t ; T)\right] x^{1}\left(t ; \tilde{x}_{0}(T), T\right) d t & +\int_{0}^{T} \bar{\Psi}(t ; T)\left[\frac{d}{d T} x^{1}\left(t ; \tilde{x}_{0}(T), T\right)\right] d t \\
& +\tilde{x}^{1}\left(t ; \tilde{x}_{0}(T), T\right) \bar{\Psi}(T ; T),
\end{aligned}
$$

provided the above exists, where we write $\bar{\Psi}_{T}(t)=\bar{\Psi}(t ; T)$ to ensure no ambiguity in the above expression. The rightmost term converges to zero. By smooth dependence on parameters, $\frac{d}{d T} x^{1}\left(t ; \tilde{x}_{0}(T), T\right)$ remains bounded in a neighbourhood of $T=T_{*}$. Let $\frac{d}{d T} x^{1}\left(t ; \tilde{x}_{0}(T), T\right) \leq D$ in this neighbourhood. 
Thus, for $T-T_{*}>0$ sufficiently small,

$$
\left|\int_{0}^{T} \bar{\Psi}(t ; T)\left[\frac{d}{d T} x^{1}\left(t ; \tilde{x}_{0}(T), T\right)\right] d t\right| \leq T \frac{K_{w} e^{r_{w} T} w_{0}(T)}{K_{w}+\left(e^{r_{w} T}-1\right) w_{0}(T)} D \rightarrow 0,
$$

as $T \rightarrow T_{*}^{+}$, since $w_{0}\left(T_{*}\right)=0$. Hence

$$
\begin{aligned}
\overline{X^{\prime}}\left(T_{*}^{+}\right) & =\lim _{T \rightarrow T_{*}^{+}} \int_{0}^{T}\left[\frac{d}{d T} \bar{\Psi}(t ; T)\right] x^{1}\left(t ; \tilde{x}_{0}(T), T\right) d t \\
& =\lim _{T \rightarrow T_{*}^{+}} \int_{0}^{T}\left[\frac{d}{d T} \bar{\Psi}(t ; T)\right] \tilde{S}_{T}(t) d t
\end{aligned}
$$

if the above exists. We have

$$
\frac{d}{d T} \bar{\Psi}(t ; T)=\frac{K_{w} q(1-q) r_{w} e^{r_{w}(t+2 T)}}{\left(e^{r_{w} t}\left(e^{r_{w} T}(1-q)-1\right)+q e^{r_{w} T}\right)^{2}} \geq 0
$$

which is jointly continuous in both of its arguments, from which it follows that that the integral $I(T)=\int_{0}^{T} \bar{\Psi}(t ; T) d t \geq 0$ exists for each $T \in\left[T^{*}, \infty\right)$, and $T \mapsto I(T)$ is continuous. Moreover, since $\bar{\Psi}\left(t ; T_{*}\right)=0$, we have $\tilde{S}_{T} \rightarrow \frac{\pi}{\mu_{H}}$ uniformly on any given compact set. By Lebesgue's dominated convergence theorem, we conclude

$$
\lim _{T \rightarrow T_{*}^{+}} \int_{0}^{T}\left[\frac{d}{d T} \bar{\Psi}(t ; T)\right] \tilde{S}_{T}(t) d t=I\left(T_{*}\right) \frac{\pi}{\mu_{H}} \geq 0,
$$

so $X^{\prime}\left(T_{*}^{+}\right)=\overline{X^{\prime}}\left(T_{*}^{+}\right)$exists and $X^{\prime}\left(T_{*}^{+}\right) \geq 0$. Define $A=\eta \frac{\beta_{H}}{H} X$. Then $A$ is $C^{1}$ and $A^{\prime}\left(T_{*}^{+}\right) \geq 0$.

If $A^{\prime}>0$ for all $T>T_{*}$, then the result holds for all $\bar{\Theta}>0$ since $A$ is invertible. Suppose then $A^{\prime}(z)=0$ for some $z_{*}>T_{*}$. Since $A^{\prime}\left(T_{*}^{+}\right) \geq 0$ and $A\left(T_{*}^{+}\right)=0$, we cannot have $A^{\prime} \leq 0$ on any interval of the form $\left(T_{*}, a\right)$, since then $A$ would be nonpositive. Hence $z_{*}$ can be chosen so that $A^{\prime}(T)>0$ for $T_{*}<T<z_{*}$. Define

$$
A_{\downarrow}(T)=\eta \frac{\beta_{H}}{H} S_{\min } \int_{0}^{T} \tilde{\Psi}_{T}(t) .
$$

Then, by $(39), A_{\downarrow}(T) \leq A(T)$, and it can be shown that $A_{\downarrow}$ is strictly increasing. 
Now define $A^{*}=A_{\downarrow}\left(z_{*}\right)$. Since $A_{\downarrow}(T) \leq A(T)$, the inequality $A^{*}<A(T)$ holds for $T>z_{*}$. Consequently, the inequality $A(T)<A^{*}$ has no solution for $T>T_{*}$. However, since $A(T)$ is increasing on $\left(T_{*}, z_{*}\right]$, there is a unique solution of the equation $A(T)=\bar{\Theta}$ for all $\bar{\Theta} \in\left(0, A^{*}\right]$, and this solution satisfies $T_{*}<T<z_{*}$. It follows from the existence proof that, for $\bar{\Theta} \leq A^{*}$, the autonomous impulsive equation has a unique periodic solution.

\section{Proof of Corollary 12.1}

The result follows directly from Theorem 11 and Theorem 12 if the transmission is by mass action. If the transmission is by standard incidence, care must be taken to ensure that we can indeed allow $h$, the disease-associated death, to be nonzero. However, it can be seen that this is a non-issue, since, by Theorem 11, the periodic solution in the system with fixed impulses is hyperbolic in the sufficiently small parameter range (where $\gamma, \delta, h$ and $\alpha$ are all small). The only part of the proof of Theorem 12 that required $h$ to be identically zero was proving that the map $X$ was surjective. Surjectivity is not required for the uniqueness of solutions for small $\bar{\Theta}$. Indeed, to prove this, we only needed to show that $X$ was $C^{1}$ at $T^{*}, X\left(T_{*}\right)=0$ and $X^{\prime}\left(T_{*}\right)=0$.

Smoothness is guaranteed by the hyperbolicity. The condition $X\left(T_{*}\right)=0$ remains valid since it depends only on boundedness of solutions and convergence of $\Psi_{T}$ to zero as $T \rightarrow T_{*}$. As for the latter, recall that the Leibniz integral formula provided the expression

$$
\begin{aligned}
X^{\prime}\left(T_{*}^{+}\right)= & \lim _{y \rightarrow T_{*}^{+}} \int_{0}^{y}\left[\frac{d}{d T} \bar{\Psi}(t ; y)\right] x^{1}\left(t ; \tilde{x}_{0}(y), y\right) d t+\int_{0}^{y} \bar{\Psi}(t ; y)\left[\frac{d}{d T} x^{1}\left(t ; \tilde{x}_{0}(y), y\right)\right] d t \\
& +\tilde{x}^{1}\left(t ; \tilde{x}_{0}(y), y\right) \bar{\Psi}(y ; y) .
\end{aligned}
$$

However, the convergence to some nonnegative number of each of these terms is provided primarily by boundedness results, in conjunction with the fact that $x^{1}\left(t ; \hat{x}_{0}\left(T_{*}\right), T_{*}\right)=\frac{\pi}{\mu_{H}}$ and $\bar{\Psi}\left(t ; T_{*}\right)=0$. None of the proofs need to be modified, since such boundedness and convergence results are preserved due to the hyperbolicity, which is guaranteed by Theorem 11 . 